\title{
HIGH-RESOLUTION HYBRID SIMULATIONS OF KINETIC PLASMA TURBULENCE AT PROTON SCALES
}

\author{
LuCa Franci $^{1,2}$, Simone Landi ${ }^{1,3}$, Lorenzo Matteini $^{1,4}$, Andrea Verdini $^{1,5}$, And Petr Hellinger ${ }^{6}$ \\ ${ }^{1}$ Dipartimento di Fisica e Astronomia, Universit degli Studi di Firenze, Largo E. Fermi 2, I-50125 Firenze, Italy; franci@arcetri.astro.it \\ ${ }_{2}$ INFN-Sezione di Firenze, Via G. Sansone 1, I-50019 Sesto F.no (Firenze), Italy \\ ${ }^{3}$ INAF-Osservatorio Astrofisico di Arcetri, Largo E. Fermi 5, I-50125 Firenze, Italy \\ ${ }_{5}^{4}$ Space and Atmospheric Physics Group, Imperial College London, London SW7 2AZ, UK \\ 5 Solar-Terrestrial Center of Excellence, Royal Observatory of Belgium, Brussels, Belgium \\ ${ }^{6}$ Astronomical Institute, AS CR, Bocni II/1401, CZ-14100 Prague, Czech Republic \\ Received 2015 June 18; accepted 2015 September 1; published 2015 October 6
}

\begin{abstract}
We investigate properties of plasma turbulence from magnetohydrodynamic (MHD) to sub-ion scales by means of two-dimensional, high-resolution hybrid particle-in-cell simulations. We impose an initial ambient magnetic field perpendicular to the simulation box, and we add a spectrum of large-scale magnetic and kinetic fluctuations with energy equipartition and vanishing correlation. Once the turbulence is fully developed, we observe an MHD inertial range, where the spectra of the perpendicular magnetic field and the perpendicular proton bulk velocity fluctuations exhibit power-law scaling with spectral indices of $-5 / 3$ and $-3 / 2$, respectively. This behavior is extended over a full decade in wavevectors and is very stable in time. A transition is observed around proton scales. At sub-ion scales, both spectra steepen, with the former still following a power law with a spectral index of $\sim-3$. A -2.8 slope is observed in the density and parallel magnetic fluctuations, highlighting the presence of compressive effects at kinetic scales. The spectrum of the perpendicular electric fluctuations follows that of the proton bulk velocity at MHD scales, and flattens at small scales. All these features, which we carefully tested against variations of many parameters, are in good agreement with solar wind observations. The turbulent cascade leads to on overall proton energization with similar heating rates in the parallel and perpendicular directions. While the parallel proton heating is found to be independent on the resistivity, the number of particles per cell, and the resolution employed, the perpendicular proton temperature strongly depends on these parameters.
\end{abstract}

Key words: plasmas - solar wind - turbulence

\section{INTRODUCTION}

Turbulence is an ubiquitous phenomenon in space and astrophysical plasmas. Although it is generally driven by violent events or instabilities at large scales, a further cascade is responsible for transferring energy via nonlinear coupling from the large injection scale to much smaller scales, through the ion and the electron characteristic regimes, where they are eventually dissipated. In situ measurements in the solar wind represent a unique opportunity to study those processes, since they provide observations on a large range of scales (see, for example, the reviews by Tu \& Marsch 1995; Matthaeus \& Velli 2011; Alexandrova et al. 2013; Bruno \& Carbone 2013). The estimated turbulent energy cascade rate (e.g., MacBride et al. 2008; Cranmer et al. 2009; Hellinger et al. 2011, 2013) is comparable to the proton heating needed to explain the nonadiabatic evolution of the solar wind plasma during its expansion (e.g., Marsch et al. 2004; Matteini et al. 2007, 2013a). This suggests that turbulence plays an active role in transferring energy from electromagnetic fields to particles and heats the solar wind plasma. However, the processes that ultimately lead to heating in a collisionless turbulent medium are still unknown.

Supporting evidence of a turbulent cascade is provided by the observed energy spectra, which exhibit a power-law behavior over a large range of scales, spanning nearly four decades in frequency. The spectral index of magnetic and kinetic spectra varies with the temperature of the solar wind streams (Grappin et al. 1990, 1991). The latter is in turn correlated with the stream speed and, although to a smaller extent, with the degree of Alfvenicity, i.e., the correlation between kinetic and magnetic fluctuations (Podesta \& Borovsky 2010; Chen et al. 2013a). However, on average, at fluidlike scales a typical Kolmogorov power law with a spectral index of $-5 / 3$ is usually observed for magnetic fluctuations, while kinetic energy spectra show a Iroshnikov-Kraichnan $-3 / 2$ scaling (Podesta et al. 2006, 2007; Salem et al. 2009; Tessein et al. 2009; Chen et al. 2011b; Wicks et al. 2011). In particular, such scaling is found to be typical of regimes with balanced turbulence, i.e., zero cross helicity (Podesta \& Borovsky 2010). In the same range of scales, a certain amount of residual energy, i.e., an excess of magnetic to kinetic energy, is typically observed, following a well-defined power-law scaling with an index of -2 (Chen et al. 2013a). The electric field spectrum is observed to follow the velocity spectrum, when measured in the solar wind frame (Chen et al. 2011a), while density fluctuations exhibit a Kolmogorov-like cascade.

In the vicinity of the ion inertial length scale, a break in the magnetic field power spectrum is observed (Beinroth \& Neubauer 1981; Goldstein et al. 1994; Leamon et al. 1998, 1999). Early observations of the spectrum of magnetic fluctuations in a restricted region above the break found a power-law scaling with a variable spectral index, ranging from -2 to -4 (e.g., Leamon et al. 1998, 1999; Bale et al. 2005; Smith et al. 2006; Alexandrova et al. 2008a, 2008b; Kiyani et al. 2009; Sahraoui et al. 2009; Chen et al. 2010; Salem et al. 2012). However, more recently, observations extended to smaller scales suggest a general convergence of the spectra toward a spectral index of -2.8 (Alexandrova et al. 2009; Kiyani et al. 2009; Sahraoui et al. 2010), or toward a power-law scaling of $-8 / 3$, exponentially damped at subelectron scales (Alexandrova et al. 2012). Magnetic 
fluctuations at sub-proton scales are also characterized by a reduction of the magnetic variance anisotropy (Podesta \& TenBarge 2012), and by an increase of the magnetic compressibility (Alexandrova et al. 2008a; Salem et al. 2012; Kiyani et al. 2013), suggesting a change in the nonlinear interactions ruling the cascade. This is partially confirmed by the measured increase of the intermittency at ion scales (Alexandrova et al. 2008a; Kiyani et al. 2009, 2013; Wu et al. 2013; Chen et al. 2014), although a clear behavior of the flatness at smaller, sub-ion scales has not yet been identified.

There are observational indications that below the ion inertial length scale, the electric field spectrum decouples from the velocity field and flattens (Bale et al. 2005; Salem et al. 2012); however, due to the high noise level, the present data do not allow us to determine the existence of a power-law scaling at sub-ion scales. Density fluctuations show a plateau just before the ion scales, while they follow a power law between the ion and the electron scales with the same spectral index as the one of the magnetic field spectrum (Chen et al. 2012, 2013b).

Properties of turbulence have been extensively analyzed by means of direct numerical simulations (DNS), employing many different methods and models. Although several features of the solar wind turbulence can be partially recovered, we are still far from a comprehensive picture. At large fluid-like scales, DNS of incompressible MHD and reduced MHD (RMHD) return a spectral index for the total energy close to $-2,-5 / 3$, or $-3 / 2$ (e.g., Maron \& Goldreich 2001; Müller et al. 2003; Müller \& Grappin 2005; Mason et al. 2008; Beresnyak \& Lazarian 2009; Perez \& Boldyrev 2009; Grappin \& Müller 2010; Lee et al. 2010; Beresnyak 2011; Boldyrev et al. 2011; Chen et al. 2011b; Perez et al. 2012). These spectral indices are associated with the different nature of the nonlinear interactions regulating the cascade and the cascade rate. Moreover, within the inertial range, a transition between different regimes can occur (Mininni \& Pouquet 2007; Verdini \& Grappin 2012). More sophisticated DNS, including other physical processes like expansion effects (Dong et al. 2014), Hall MHD (e.g., Matthaeus et al. 2003; Gómez et al. 2008; Shaikh \& Shukla 2009; Shaikh \& Zank 2009), reduced Hall MHD (Gómez et al. 2013), gyrokinetic (Howes et al. 2008; TenBarge et al. 2013), and hybrid particle-in-cell (PIC) simulations (Vasquez \& Markovskii 2012) all produce spectral indices consistent with $-5 / 3$. Regardless, the restricted width of the inertial range prevents firm conclusions.

As far as the small kinetic scales are concerned, DNS including proton and electron physics return a qualitatively unified picture. At sub-proton scales, they reproduce an increase of the ratio of the electric to magnetic power, together with a flattening of the electric field spectrum (e.g., Dmitruk \& Matthaeus 2006; Howes et al. 2008, 2011; Servidio et al. 2012; Gómez et al. 2013; Perrone et al. 2013; Parashar et al. 2014; Passot et al. 2014; Valentini et al. 2014; Servidio et al. 2015), and a transition to a steeper spectrum for the magnetic field power near the ion scales (e.g., Matthaeus et al. 2003; Dmitruk \& Matthaeus 2006; Parashar et al. 2010; Servidio et al. 2012; Vasquez \& Markovskii 2012; Rodriguez Imazio et al. 2013; Valentini et al. 2014). However, a unique spectral index cannot be identified for the magnetic field spectrum at proton scales. Early works in Hall MHD (Shaikh \& Shukla 2009; Martin et al. 2013), Electron-MHD (Biskamp et al. 1999; Ng et al. 2003; Cho \& Lazarian 2004, 2009; Shaikh 2009), and gyrokinetic (Howes et al. 2008) reported a spectral index of $-7 / 3$ for the magnetic field at sub-ion scales. More recently, steeper spectra have also been observed: a spectral index of -2.8 in gyrokinetic (Howes et al. 2011; TenBarge \& Howes 2013; TenBarge et al. 2013) and finite Larmor radiusLandau fluid simulations (Passot et al. 2014), or a $-8 / 3$ power law both in three-dimensional (3D) electron-MHD (Meyrand \& Galtier 2013) and in strong kinetic-Alfvén turbulence (Boldyrev \& Perez 2012). Magnetic spectral indices in between about -2.6 and -3 have also been observed in full PIC simulations (e.g., Camporeale \& Burgess 2011; Chang et al. 2011; Wan et al. 2012; Karimabadi et al. 2013; Wu et al. 2013).

In situ measurements of the proton velocity distribution functions show the presence of an ubiquitous temperature anisotropy between the direction parallel and perpendicular to the mean magnetic field (Marsch et al. 1982; Hellinger et al. 2006), and a non-adiabatic evolution of the solar wind plasma during its expansion (Marsch et al. 2004; Matteini et al. 2007, 2013a), thus suggesting, as already mentioned, an active role played by the turbulence in exchanging energy between fields and particles. Hybrid PIC simulations have shown an overall (macroscopic) collisionless proton heating, with signatures of a preferential proton heating in the perpendicular direction with respect to the ambient mean magnetic field (e.g., Parashar et al. 2009; Markovskii et al. 2010; Markovskii \& Vasquez 2011; Vasquez \& Markovskii 2012; Verscharen et al. 2012; Parashar et al. 2014). Vlasov-hybrid simulations suggest that non-Maxwellian kinetic effects, such as temperature anisotropies, can be produced by the turbulence, mostly concentrated in regions near and around the peaks of the current density (Servidio et al. 2012; Perrone et al. 2014; Valentini et al. 2014; Servidio et al. 2015).

In our previous work (Franci et al. 2015, hereafter called Letter 1), we presented results from a high-resolution hybrid (fluid electrons, PIC protons) two-dimensional (2D) simulations of turbulence. The spectra of various fluctuations (magnetic, kinetic, density, and electric field), along with the magnetic compressibility and the non-dimensional ratio of the density and the magnetic fluctuations, simultaneously matched several features observed in the solar wind. In particular, for the magnetic field we showed that high-resolution hybrid simulations, although limited to a 2D geometry, are able to capture the nonlinear dynamics at fluid-like MHD scales and at sub-proton scales, both within the same numerical domain. In this paper, we analyze in further detail the spectral properties of several fields, also showing their stability with time. Moreover, we investigate the shape of the electric field spectrum by estimating the separated contributions from different terms in the generalized Ohm's law. Finally, we study the proton temperature anisotropy and the proton heating, also quantifying the dependence from the resistivity coefficient and the number of particles-per-cell (ppc) employed in the simulations.

The paper is organized as follows. In Section 2, we describe the numerical setup employed, define the physical units and normalizations in the code, and provide the parameters of our initial conditions. In Section 3, we describe the results of the simulations performed. In Section 4, we validate such results by investigating the importance of a careful choice of some relevant numerical parameters. Finally, in Section 5, we summarize the achievements of our simulations and discuss 
Table 1

List of Simulations and Their Relevant Parameters

\begin{tabular}{lcccc}
\hline \hline Run & $\begin{array}{c}\Delta x \\
\left(d_{\mathrm{p}}\right)\end{array}$ & $\begin{array}{c}L_{\text {box }} \\
\left(d_{\mathrm{p}}\right)\end{array}$ & $\begin{array}{c}\eta \\
\left(4 \pi / \omega_{\mathrm{p}}\right)\end{array}$ & $\mathrm{ppc}$ \\
\hline A & 0.125 & 256 & $5 \times 10^{-4}$ & 8000 \\
\hline B & 0.125 & 256 & $5 \times 10^{-4}$ & 4000 \\
C & 0.125 & 256 & $5 \times 10^{-4}$ & 2000 \\
D & 0.125 & 256 & $5 \times 10^{-4}$ & 1000 \\
E & 0.125 & 256 & $5 \times 10^{-4}$ & 500 \\
\hline F & 0.125 & 256 & $1 \times 10^{-4}$ & 8000 \\
G & 0.125 & 256 & $1 \times 10^{-3}$ & 8000 \\
\hline H & 0.250 & 512 & $1 \times 10^{-3}$ & 8000 \\
I & 0.500 & 1024 & $2 \times 10^{-3}$ & 8000 \\
\hline
\end{tabular}

them in the framework of both observational and previous numerical and theoretical studies.

\section{NUMERICAL SETUP AND INITIAL CONDITIONS}

We make use of a 2D hybrid code, where electrons are considered as a massless charge-neutralizing fluid with a constant temperature, whereas ions are described by a PIC model and are advanced by the Boris' scheme, which requires the fields to be known at a half time step ahead of the particle velocities. This is achieved by advancing the current density to this time step with only one computational pass through the particle data at each time step (Matthews 1994).

The characteristic temporal and spatial units used in this model are the inverse proton gyrofrequency $\Omega_{\mathrm{p}}^{-1}=$ $\left(e B_{0} / m_{\mathrm{p}} c\right)^{-1}$ and the proton inertial length $d_{\mathrm{p}}=c / \omega_{\mathrm{p}}$, respectively, with $\omega_{\mathrm{p}}=\left(4 \pi n e^{2} / m_{\mathrm{p}}\right)^{1 / 2}$ being the proton plasma frequency. Magnetic fields are expressed in units of the magnitude of the ambient magnetic field, i.e., $B_{0}$, while velocities are expressed in units of the Alfvén velocity, i.e., $v_{\mathrm{A}}=B_{0} /\left(4 \pi n m_{\mathrm{p}}\right)^{1 / 2}$. The plasma beta for a given plasma species, protons (p) or electrons (e), is $\beta_{\mathrm{p}, \mathrm{e}}=8 \pi n K_{\mathrm{B}} T_{\mathrm{p}, \mathrm{e}} / B_{0}^{2}$. Quantities and symbols used in these definitions are the speed of light, $c$, the number density, $n$, which is assumed to be equal for proton and electrons $\left(n_{\mathrm{p}}=n_{\mathrm{e}}=n\right)$, the magnitude of the electronic charge, $e$, the proton mass, $m_{\mathrm{p}}$, the Boltzmann's constant, $K_{\mathrm{B}}$, and the proton and electron temperatures, $T_{\mathrm{p}, \mathrm{e}}$.

The 2D computational domain lies in the $(x, y)$ plane, while the ambient magnetic field $\boldsymbol{B}_{0}$ is along the $z$-direction. Accordingly, each field $\Psi$ will be decomposed in its perpendicular (in-plane) component, $\Psi_{\perp}$, and its parallel (outof-plane, along $z$ ) component, $\Psi_{\|}$, with respect to $\boldsymbol{B}_{0}$. The only exceptions will be the proton beta and temperature, for which $\perp$ and $\|$ will denote directions with respect to the local magnetic field.

The adopted simulation box is a $2048^{2}$ square grid. We tested different resolutions $\left(\Delta x=\Delta y=0.5,0.25\right.$ and $\left.0.125 d_{\mathrm{p}}\right)$ and consequently different box sizes $\left(L_{\mathrm{box}}=1024,512\right.$ and $\left.256 d_{\mathrm{p}}\right)$ , as well as different numbers of ppc, ranging from 500 to 8000 (see Table 1). The time step for the particle advance is $\Delta t=0.025 \Omega_{\mathrm{p}}^{-1}$, whereas the magnetic field $\boldsymbol{B}$ is advanced with a smaller time step, $\Delta t_{\mathrm{B}}=\Delta t / 10$.

Initially, we assume a uniform number density $n=1$, a proton parallel beta $\beta_{\mathrm{p} \|}=0.5$, and a temperature anisotropy
$A_{\mathrm{p}}=T_{\mathrm{p} \perp} / T_{\mathrm{p} \|}=1$. Electrons are isotropic, with $\beta_{\mathrm{e}}=0.5$. We add an initial spectrum of magnetic and velocity fluctuations in the $(x, y)$ plane, composed of modes with $-0.2<k_{x, y}<0.2$ in each direction and random phases. These initial fluctuations are characterized by energy equipartition and vanishing correlation between kinetic and magnetic fluctuations, and their global amplitude is $B^{\mathrm{rms}} \sim 0.24$. Hereafter,

$$
\Psi^{\mathrm{rms}}=\left(\left\langle\Psi^{2}\right\rangle-\langle\Psi\rangle^{2}\right)^{1 / 2}
$$

will denote the root mean square value (rms) of a quantity $\Psi$, with $\langle\Psi\rangle$ being its space-averaged value over the entire $2 \mathrm{D}$ simulation domain. The initial magnetic fluctuations can be expressed in the form

$$
\begin{aligned}
\boldsymbol{B}_{\perp}(x, y)= & \frac{1}{2} \sum_{k_{x}, k_{y}}\left[\boldsymbol{B}_{\perp}\left(k_{x}, k_{y}\right)\right. \\
& \left.\times \exp \left(i\left(k_{x} x+k_{y} y+\phi\left(k_{x}, k_{y}\right)\right)\right)+\text { c.c. }\right] .
\end{aligned}
$$

The initial bulk velocity fluctuations $\boldsymbol{u}_{\perp}(t=0)$ are assumed to have the same form as in Equation (2), with different random phases.

We introduce two dimensionless quantities, i.e., the normalized cross helicity, $\sigma_{\mathrm{C}}$, and the normalized residual energy, $\sigma_{\mathrm{R}}$ :

$$
\begin{aligned}
& \sigma_{\mathrm{C}}(x, y)=\frac{2 \boldsymbol{u} \cdot \boldsymbol{B}}{|\boldsymbol{u}|^{2}+|\boldsymbol{B}|^{2}}, \\
& \sigma_{\mathrm{R}}(x, y)=\frac{|\boldsymbol{u}|^{2}-|\boldsymbol{B}|^{2}}{|\boldsymbol{u}|^{2}+|\boldsymbol{B}|^{2}},
\end{aligned}
$$

which define the 2D geometry of the fluctuations. With the initial conditions we chose for the initial magnetic and bulk velocity fluctuations, $\sigma_{\mathrm{C}}$ and $\sigma_{\mathrm{R}}$ are both statistically very close to zero, even though they are not actually zero anywhere in the $(x, y)$ plane.

A non-zero resistivity has been introduced in order to guarantee a satisfactory conservation of the total energy, with no claim to model any realistic physical process. Its value has been empirically fine-tuned by running different simulations (see Table 1). Further details about this point will be provided in Section 4.

\section{RESULTS}

We performed nine different simulations. Their main parameters are listed in Table 1. A label is assigned to each run in the first column, while in the other columns we report, from left to right: the spatial resolution, $\Delta x(=\Delta y)$, the length of the simulation box, $L_{\mathrm{box}}$, the value of the resistivity coefficient, $\eta$, and the number of ppc.

Run A employs the best spatial resolution, $\Delta x=\Delta y=0.125 d_{\mathrm{p}}$, and the highest number of particles, i.e., $8000 \mathrm{ppc}$, corresponding to more than $3 \times 10^{10}$ particles in the whole simulation domain. The resistive coefficient has been fine-tuned and set to the value $\eta=5 \times 10^{-4}$, in units of $4 \pi \omega_{\mathrm{p}}^{-1}$. In Sections 3.1 and 3.2 we will provide a detailed and quantitative analysis of the data produced by this run. The remaining simulations were performed in order to validate these results and to investigate the effects of the number of ppc (Runs B-E, see Table 1), the resistivity (Runs F-G), and the 


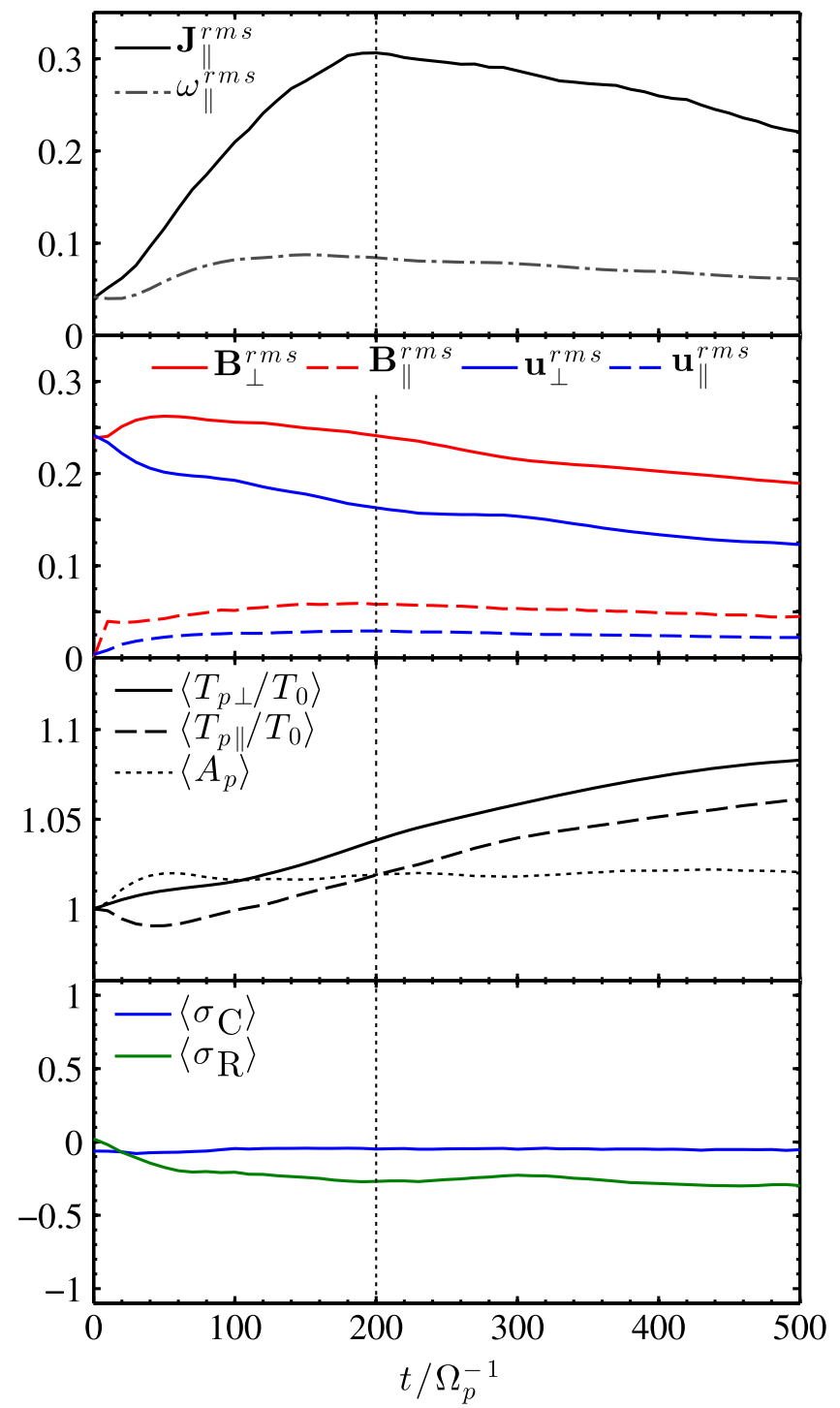

Figure 1. Time evolution of space-averaged quantities. From top to bottom: the rms out-of-plane current density, $\boldsymbol{J}_{\|}$, and the rms out-of-plane vorticity, $\boldsymbol{\omega}_{\|}$(first panel); the rms perpendicular $\boldsymbol{B}_{\perp}$ and parallel $\boldsymbol{B}_{\|}$magnetic fluctuations, and the rms perpendicular $\boldsymbol{u}_{\perp}$ and parallel $\boldsymbol{u}_{\|}$velocity fluctuations (second panel); the mean values of the normalized perpendicular and parallel proton temperatures, $T_{\mathrm{p} \perp} / T_{0}$ and $T_{\mathrm{p} \|} / T_{0}$, and of the proton temperature anisotropy, $A_{\mathrm{p}}$ (third panel); the mean values of the normalized cross helicity, $\sigma_{\mathrm{C}}$, and of the normalized residual energy, $\sigma_{\mathrm{R}}$ (fourth panel). In all panels, a vertical black dotted line marks the time of the maximum turbulent activity, i.e., $t=200 \Omega_{\mathrm{p}}^{-1}$.

spatial resolution (Runs H-I). Their results will be discussed in Section 4.

\subsection{Temporal and Spatial Evolution}

In Figure 1, the time evolution of a few quantities is shown up to $500 \Omega_{\mathrm{p}}^{-1}$. The initial nonlinear time associated with the maximum injection scale, i.e., $k^{\text {inj }} \sim 0.2 d_{\mathrm{p}}^{-1}$, can be estimated as $t_{\mathrm{NL}} \sim\left[k^{\mathrm{inj}} \delta u_{k^{\mathrm{inj}}}\right]^{-1} \sim 20 \Omega_{\mathrm{p}}^{-1}$, and corresponds to the minor ticks of the $x$ axis. The total length of the simulation is approximately $25 t_{\mathrm{NL}}$.

In the first panel, from top to bottom, we report the rms outof-plane current density, $\boldsymbol{J}_{\|}$, and the rms out-of-plane vorticity, $\boldsymbol{\omega}_{\|}$. The current increases quite rapidly, attains its maximum value just before $t \sim 200 \Omega_{\mathrm{p}}^{-1}$, and then slowly decreases.
Since it represents a good indicator of the level of turbulent activity (Mininni \& Pouquet 2009), we choose to perform a detailed analysis at $t \sim 200 \Omega_{\mathrm{p}}^{-1}$, when the turbulence is expected to be fully developed. A vertical black dotted line marks this time in all four panels. The vorticity also increases quite rapidly, reaching an earlier and lower maximum value, and then it decreases extremely slowly.

The second panel of Figure 1 shows the rms perpendicular $\boldsymbol{B}_{\perp}$ and parallel $\boldsymbol{B}_{\|}$magnetic fluctuations (red lines), and the rms perpendicular $\boldsymbol{u}_{\perp}$ and parallel $\boldsymbol{u}_{\|}$velocity fluctuations (blue lines). Perpendicular and parallel components are drawn with solid and dashed lines, respectively. $\boldsymbol{B}_{\perp}$ exhibits a small increase until $t \sim 40 \Omega_{\mathrm{p}}^{-1}$, and then it decreases quite smoothly. On the other hand, $\boldsymbol{u}_{\perp}$ decreases with a similar trend, but without showing any initial growth. This indicates that the turbulence is fed by the perpendicular components of both the magnetic and the velocity fluctuations, whose energy decreases slowly and sustains the cascade for the whole evolution. Contextually, the parallel components of both the magnetic and the velocity fluctuations rapidly originate from compressive effects, remaining much smaller than their perpendicular counterparts throughout the simulation.

In the third panel of Figure 1, we report the space-averaged parallel and perpendicular proton temperatures, normalized to the initial value $T_{0},\left\langle T_{\mathrm{p} \|} / T_{0}\right\rangle$ and $\left\langle T_{\mathrm{p} \perp} / T_{0}\right\rangle$, respectively, together with the space-averaged proton temperature anisotropy, $\left\langle A_{\mathrm{p}}\right\rangle=\left\langle T_{\mathrm{p} \perp} / T_{\mathrm{p} \|}\right\rangle$. We recall here that $T_{\|}$and $T_{\perp}$ are defined with respect to the local magnetic field. Since $\left\langle A_{\mathrm{p}}\right\rangle=1$ is imposed at $t=0, T_{\mathrm{p} \|}$ and $T_{\mathrm{p} \perp}$ share the same initial value, $T_{0}$. In the very first part of the evolution, the former shows a little and sudden decrease, after which both increase with almost the same rate. The parallel and perpendicular energy gains, at the end of the simulation, i.e., at $t=500 \Omega_{\mathrm{p}}^{-1}$, are approximately $6 \%$ and $8 \%$, respectively. This small excess of perpendicular energy quickly arises within $\sim 2 t_{\mathrm{NL}}$, and it is preserved throughout the simulation, with the temperature anisotropy reaching a value $\left\langle A_{\mathrm{p}}\right\rangle \sim 1.02$ in correspondence to the maximum turbulent activity, and then remaining quite constant until the end of the simulation. A detailed discussion about the proton heating will be further provided in Section 4.2.

Lastly, in the bottom panel of Figure 1, we show the spaceaveraged values of the normalized cross helicity, $\sigma_{\mathrm{C}}$, and of the residual energy, $\sigma_{\mathrm{R}}$, (see Equations (3) and (4)). The former is very close to zero at the beginning of the simulation (as a result of the initially imposed random phases spectra), and it tends to maintain this value until the end. The latter, instead, decreases from zero to about -0.3 in very few nonlinear times $t_{\mathrm{NL}}$, showing a global excess of the magnetic energy over the kinetic energy. These asymptotic values are reached very quickly as a consequence of the relaxation from the initial random relative orientation of the velocity and the magnetic fluctuations toward a strongly aligned state. Despite the steady time evolution of their space-averaged values, both $\sigma_{\mathrm{C}}$ and $\sigma_{\mathrm{R}}$ appear very patchy when looking at the spatial distribution throughout the 2D computational domain (not shown), exhibiting quite a wide excursion from -1 to 1 between different albeit close regions.

Summarizing the time evolution of all the above-mentioned quantities, we can divide the evolution of the system in three different stages.

1. A rapid readjustment and relaxation of the initial conditions, occurring within $t \lesssim 40 \Omega_{\mathrm{p}}^{-1} \sim 2 t_{\mathrm{NL}}$. 
$\left|\mathbf{B}_{\perp}\right|^{2}$
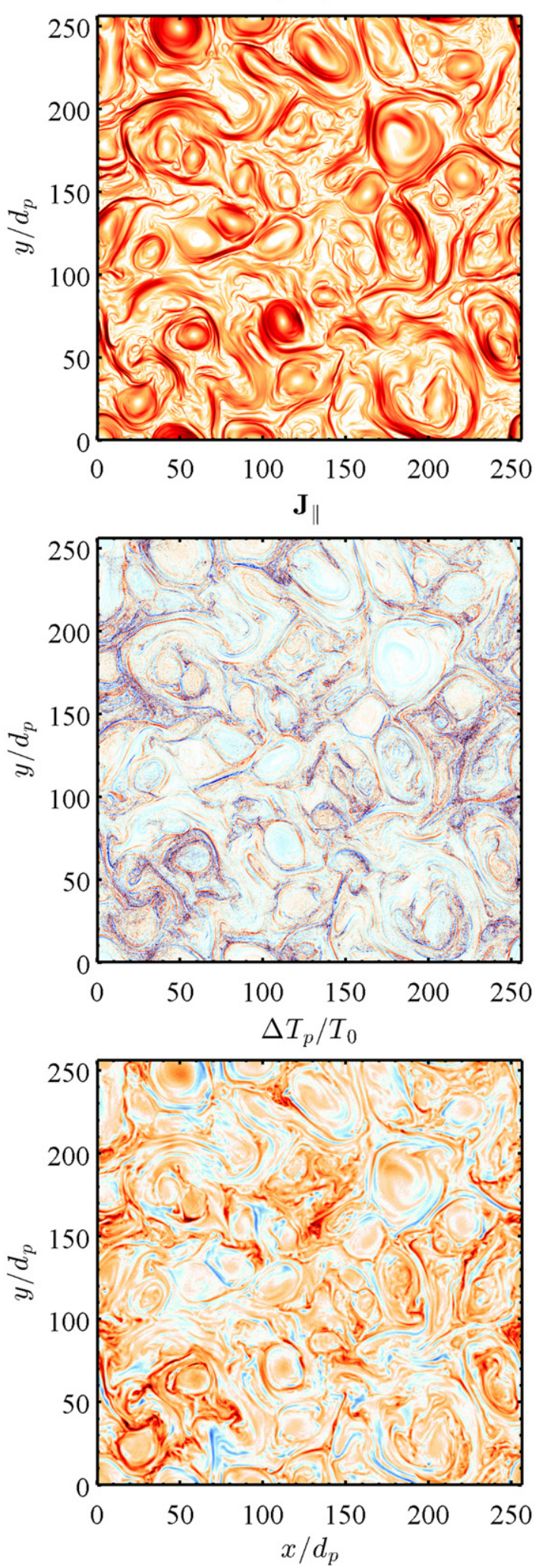

$\left|\mathbf{u}_{\perp}\right|^{2}$
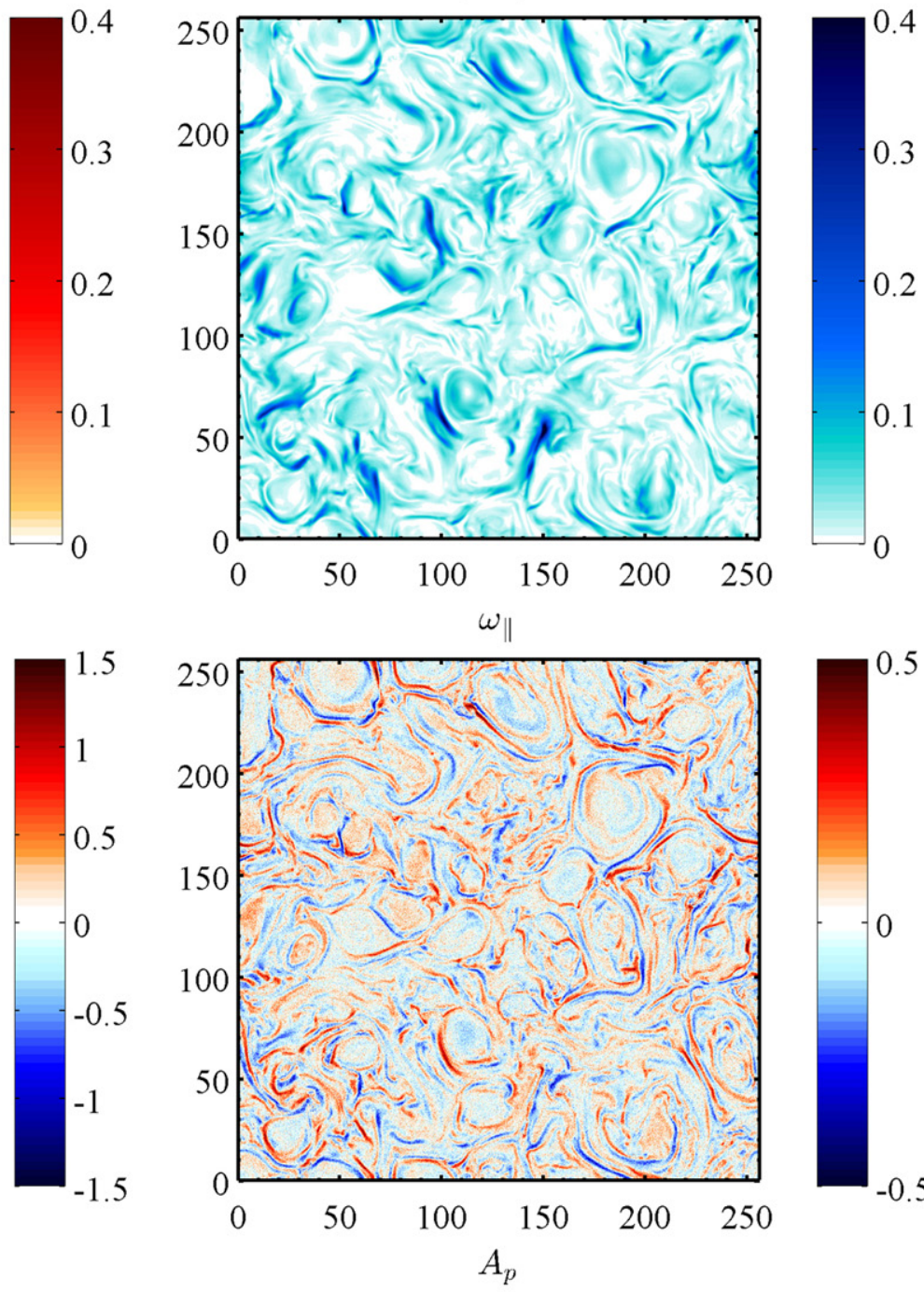

0.5
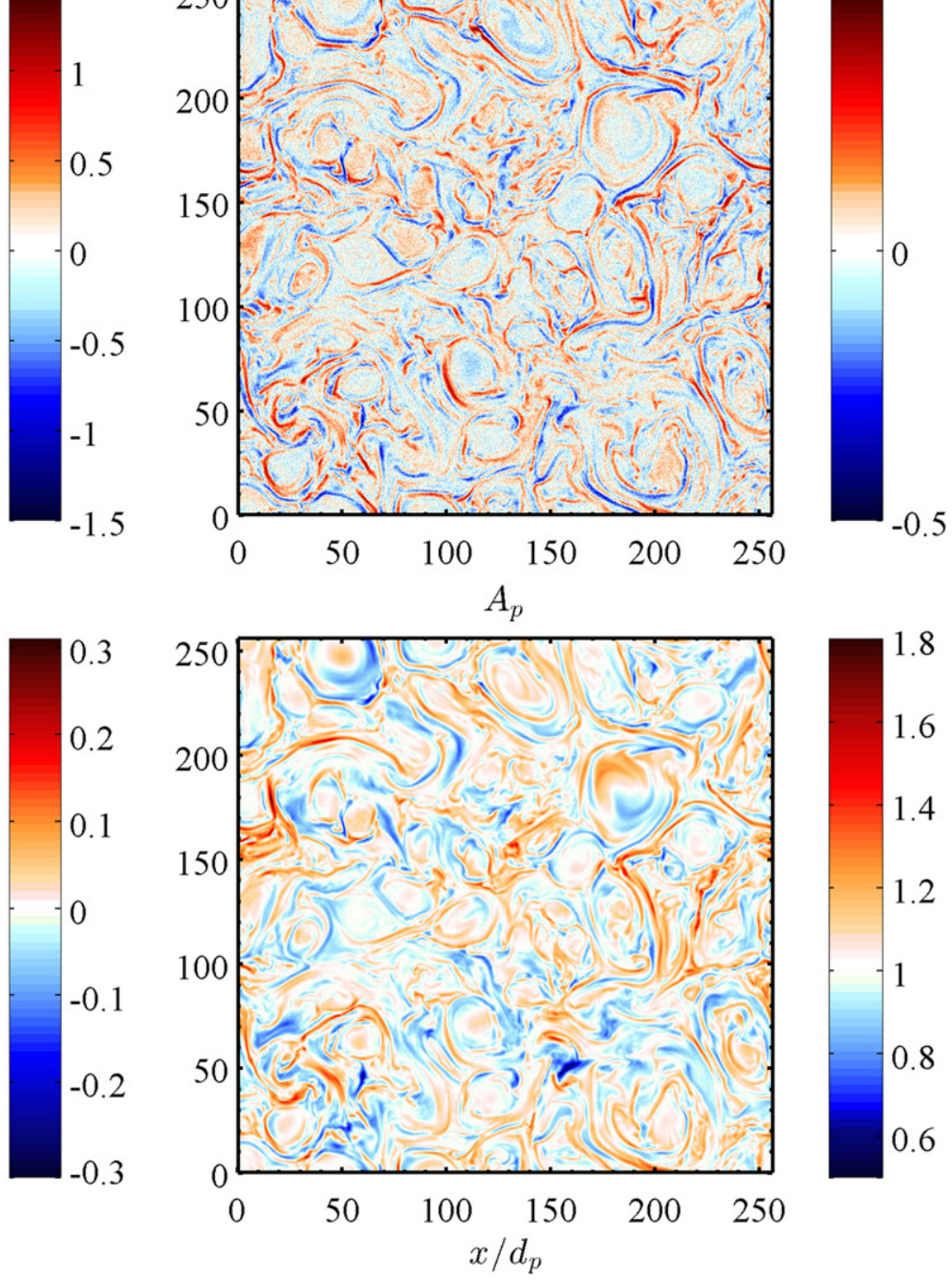

1.6

1.4

1.2

$-1$

0.8

0.6

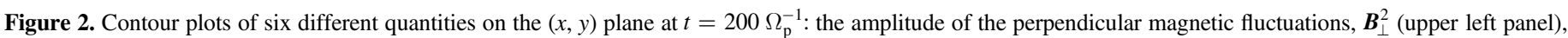

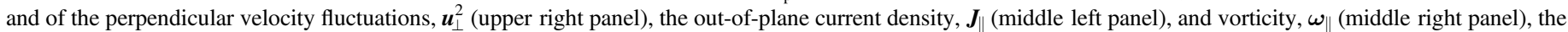
proton temperature variation normalized to the initial temperature, $\Delta T_{\mathrm{p}} / T_{0}$ (bottom left panel), and the proton temperature anisotropy, $A_{\mathrm{p}}$ (bottom right panel). 

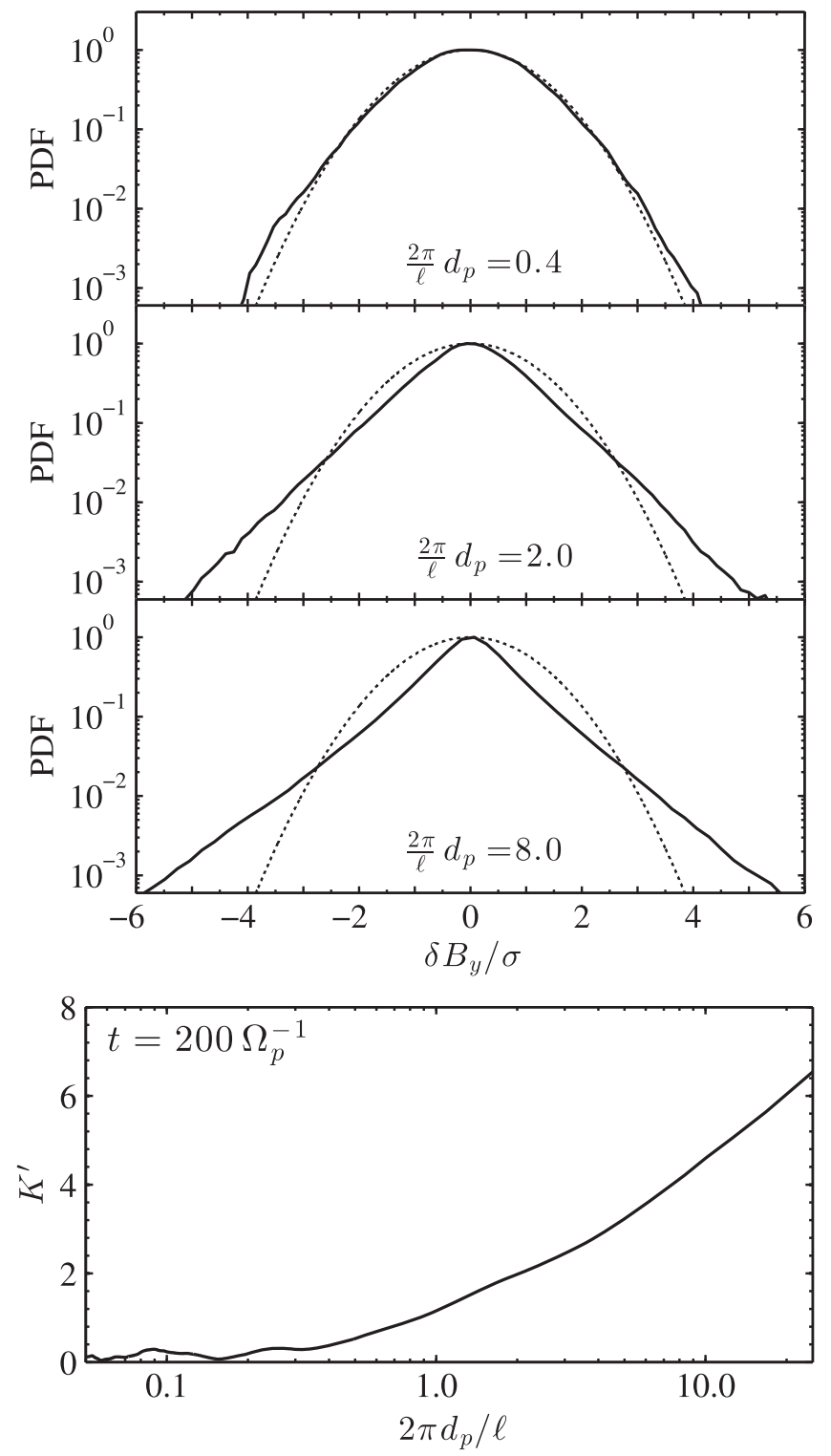

Figure 3. Top panel: PDFs of the increments of the perpendicular magnetic field component $B_{y}$ along $x$ at $t=200 \Omega_{\mathrm{p}}^{-1}$, corresponding to $2 \pi d_{\mathrm{p}} / \ell=0.4$, $2 \pi d_{\mathrm{p}} / \ell=2$ and $2 \pi d_{\mathrm{p}} / \ell=8$ (from top to bottom). In each panel, a Gaussian function with the same variance is plotted with a dashed line as a reference. Bottom panel: excess kurtosis of the same quantity computed at the same time.

2. The onset of a turbulent cascade, fed by the perpendicular magnetic and velocity fluctuations, involving larger and larger scales on times of the order of $t \sim 200 \Omega_{\mathrm{p}}^{-1} \sim 10 t_{\mathrm{NL}}$.

3. A decaying phase with slow and smooth variations of all rms quantities, during which the turbulence is fully developed and further sustained until at least $t \sim 500 \Omega_{\mathrm{p}}^{-1}$, corresponding to $\sim 25 t_{\mathrm{NL}}$.

Figure 2 shows isocontours of six different quantities in the whole simulation domain, all computed at $t=200 \Omega_{\mathrm{p}}^{-1}$. In the upper left panel, we report the magnitude of the perpendicular magnetic fluctuations, $\left|\boldsymbol{B}_{\perp}\right|^{2}$, showing the presence of coherent structures in the magnetic field, i.e., vortices and magnetic islands, embedded in a much more chaotic environment where stretched and twisted shapes emerge. In the upper right panel, the magnitude of the perpendicular velocity fluctuations, $\left|\boldsymbol{u}_{\perp}\right|^{2}$, is shown to exhibit qualitatively the same kind of structures, but with lower intensity and much lower gradients. In some regions, high values of $\left|\boldsymbol{u}_{\perp}\right|^{2}$ correspond to high values of $\left|\boldsymbol{B}_{\perp}\right|^{2}$, while in other regions the opposite situation holds. In the middle left panel, we show the out-of-plane current density, $J_{\|}=(\nabla \times \boldsymbol{B})_{\|}$. Many thin current sheets form since the very first phase of the evolution, mostly around and in between vortices. Once formed, each current sheet is quickly disrupted into smaller and smaller pieces, contributing to the generation of smaller scale structures. At the time of maximum turbulent activity, this results in the articulated pattern shown here. In the middle right panel, the out-of-plane vorticity, $\boldsymbol{\omega}_{\|}=(\nabla \times \boldsymbol{u})_{\|}$, is shown. It exhibits a structure similar to $\boldsymbol{J}_{\|}$, with many thin layers, whose shape is, however, much more defined and clean with respect to $\boldsymbol{J}_{\|}$. Peaks of $\boldsymbol{\omega}_{\|}$and peaks of $\boldsymbol{J}_{\|}$occupy approximately the same regions. In the bottom left panel, we report the proton temperature variation with respect to the initial proton temperature, $\Delta T_{\mathrm{p}} / T_{0}=\left(T_{\mathrm{p}}-T_{0}\right) / T_{0}$, where $T_{\mathrm{p}}=\left(2 T_{\mathrm{p} \perp}+T_{\mathrm{p} \|}\right) / 3$ is the average proton temperature measured at $t=200 \Omega_{\mathrm{p}}^{-1}$. Regions where $\Delta T_{\mathrm{p}}$ is locally both negative or positive are clearly present, and a resulting global proton temperature enhancement can be observed, as already inferred from Figure 1. Interestingly, areas where a proton temperature enhancement occurs are located in the vicinity of current sheets (see Servidio et al. 2012). The presence of proton temperature anisotropies in correspondance with current sheets may have an effect on their stability (Matteini et al. 2013b; Gingell et al. 2015). A more detailed analysis shows that strong currents exhibit a complex evolution, which involves splitting/ dissociation and leads to a relevant proton energization.

In the bottom right panel, the proton temperature anisotropy, $A_{\mathrm{p}}$, is shown. We observe a wide excursion between very close areas, the perpendicular proton temperature $T_{\mathrm{p} \perp}$ ranging from about half and almost twice the parallel one. Therefore, there is a strong local reshaping of particle distributions, leading to both perpendicular and parallel anisotropies (Servidio et al. 2014). Nevertheless, as can be inferred from Figure 1, the relative difference between $\left\langle T_{\mathrm{p} \perp}\right\rangle$ and $\left\langle T_{\mathrm{p} \|}\right\rangle$ is about $2 \%$ at $t=200 \Omega_{\mathrm{p}}^{-1}$, meaning that globally no preferential enhancement along the perpendicular or parallel direction is achieved. More generally, when starting with a proton temperature anisotropy other than 1 , a spread of $A_{\mathrm{p}}$ is quickly generated around its initial value (Hellinger et al. 2015).

The small-scale coherent structures which have emerged by the time of maximum turbulent activity, already observed in Figure 2, can be related to the phenomenon of intermittency since they are able to induce departures from self-similarity and enhanced dissipation. In order to look for intermittency in our data, we examine the non-Gaussian behavior of the probability density function (PDF) of an MHD primitive variable. In particular, we compute the PDFs at $t=200 \Omega_{\mathrm{p}}^{-1}$ by taking increments of one of the perpendicular magnetic field components, i.e., $\boldsymbol{B}_{y}$, along the other perpendicular direction, i.e., $x$, for three different spatial separations, $\ell$. In the top three panels of Figure 3, we show three PDFs, computed for $2 \pi d_{\mathrm{p}} / \ell=0.4$, which is approximatively in the middle of the inertial range (upper panel), $2 \pi d_{\mathrm{p}} / \ell=2$, which is the scale corresponding to the ion spectral break (middle panel), and $2 \pi d_{\mathrm{p}} / \ell=8$, which is well inside the kinetic range (bottom panel). A Gaussian function with the same variance is plotted with a dashed line in each panel as a reference. The distribution 
of magnetic fluctuations is clearly different at different scales: it is closer to a normal distribution at very large scales, it shows a significant deviation at intermediate scales, and it displays very extended tails at small scales. In order to quantify the level of intermittency, we compute the fourth central moment $K$ (or kurtosis) of the distributions. In the bottom panel of Figure 3, we show the excess kurtosis $K^{\prime}=K-3$, computed from the increments of $\boldsymbol{B}_{y}$ along $x$, as a function of $2 \pi d_{\mathrm{p}} / \ell$, again at $t=200 \Omega_{\mathrm{p}}^{-1}$. This quantity is clearly very close to zero up to the injection scale, i.e., $k_{\perp} d_{\mathrm{p}} \lesssim 0.2$, and the it steadily increases through the inertial range and down to sub-proton scales.

\subsection{Spectral Properties}

Since the small-scale structures shown in Figure 2 exhibit random orientations, and therefore the 2D spectra of all fluctuations can be assumed statistically isotropic, we can perform a quantitative analysis of the turbulent cascade by computing the omnidirectional spectra. These are defined as

$$
P_{\Psi}\left(k_{\perp}\right) \equiv \delta \Psi^{2}\left(k_{\perp}\right) / k_{\perp}=\sum_{\left|\boldsymbol{k}_{\perp}\right|=k_{\perp}} \hat{\Psi}_{2 \mathrm{D}}^{2}\left(\boldsymbol{k}_{\perp}\right),
$$

where $\hat{\Psi}$ are the Fourier coefficients of a given quantity $\Psi$, and $\delta \Psi\left(k_{\perp}\right)$ is the amplitude of the fluctuation $\Psi$ at the scale $k_{\perp}$.

In Figure 4, we show the spectra of the perpendicular magnetic and velocity fluctuations, drawn with red and blue solid lines, respectively, at $t=200 \Omega_{\mathrm{p}}^{-1}$. We clearly observe two power-law ranges, separated by a smooth spectral break at a scale of the order of the the proton inertial length, $k_{\perp} d_{\mathrm{p}} \sim 2$. In Letter 1, we showed the spectra of the total magnetic and velocity fluctuations, which exhibit a very similar behavior, since the perpendicular components are the dominant ones for both field.

In particular, in the inertial range the spectrum of the perpendicular magnetic fluctuations follows a Kolmogorov $k_{\perp}^{-5 / 3}$ power-law scaling over a full decade in wavenumber, approximately between $k_{\perp} d_{\mathrm{p}}=0.1$ and 2 . Simultaneously, the perpendicular proton bulk velocity fluctuations exhibit a less steep slope, with an Iroshnikov-Kraichnan scaling of $k_{\perp}^{-3 / 2}$, over a little less than a decade, in the range $0.2 \lesssim k_{\perp} d_{\mathrm{p}} \lesssim 1$. Moreover, an excess of magnetic energy over kinetic energy is observed, coherently with the negative value of the normalized residual energy $\sigma_{\mathrm{R}}$ already shown in the bottom panel of Figure 1.

At kinetic scales, the spectra of both fields steepen, due to the presence of both kinetic and dissipative (resistive) effects. The spectrum of $\boldsymbol{u}_{\perp}$ quickly drops with an exponential trend above $k_{\perp} d_{\mathrm{p}} \sim 1$, until it clearly saturates to the noise level due to the finite number of ppc, corresponding to the spectrum at $t=0$. The spectrum of the magnetic fluctuations, on the contrary, continue to follow a power-law scaling also at subproton scales, although with a steeper spectral index of the order of -3 . For $k_{\perp} d_{\mathrm{p}} \gtrsim 10, P_{\boldsymbol{B}_{\perp}}$ does not show an exponential damping, as one would expect for resistive dissipation, but a small increase instead, since the adopted resistive coefficient is slightly smaller than the optimal value.

As discussed, the maximum level of turbulent activity occurs at $t \sim 200 \Omega_{\mathrm{p}}^{-1}$, which is about 10 times the initial nominal nonlinear time $t_{\mathrm{NL}}$. This can be explained by the fact that at $t=0$ we inject energy through several modes within the range

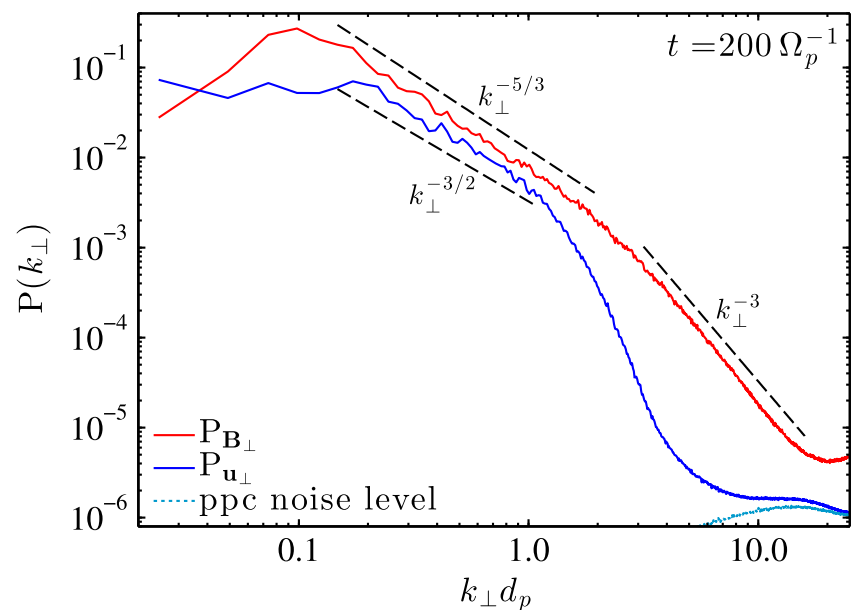

Figure 4. Power spectra of the perpendicular magnetic and velocity fluctuations, $\boldsymbol{B}_{\perp}$ (red solid line) and $\boldsymbol{u}_{\perp}$ (blue solid line), respectively. Power laws with different spectral indices are additionally shown as black dashed lines as a reference.

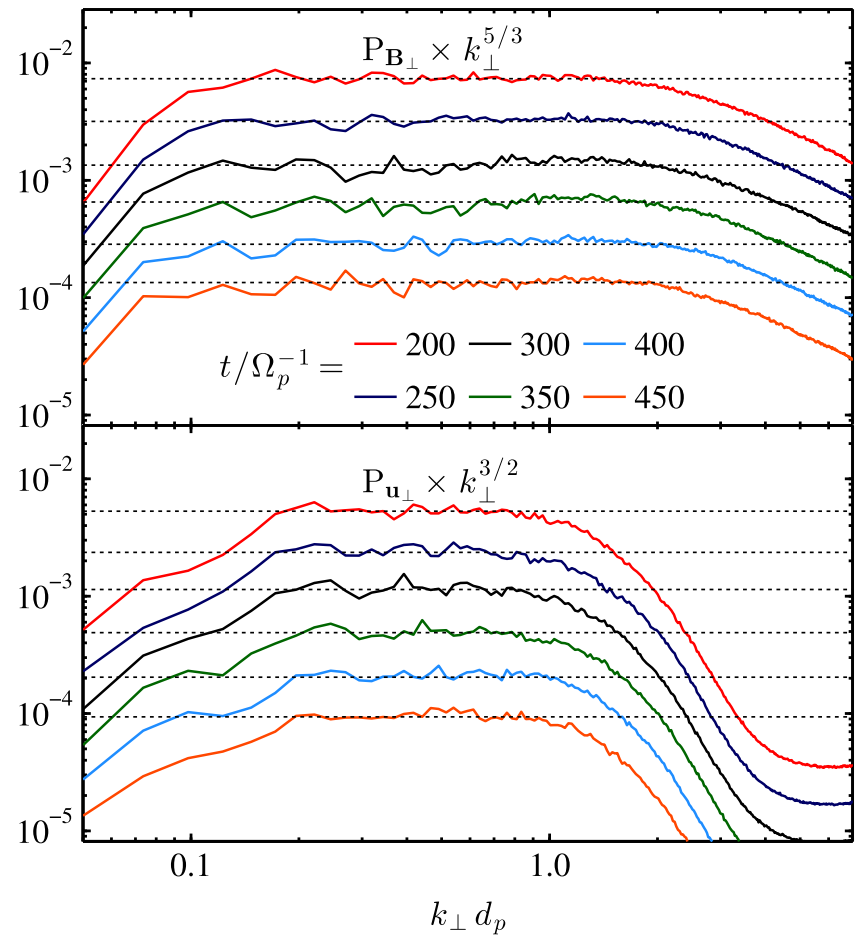

Figure 5. Top panel: power spectra of the perpendicular magnetic fluctuations, $\boldsymbol{B}_{\perp}$, compensated by $k_{\perp}^{5 / 3}$. Each of them is the time-average in the interval $\left[\tilde{t}-20 \Omega_{\mathrm{p}}^{-1}, \tilde{t}+20 \Omega_{\mathrm{p}}^{-1}\right]$, where $\tilde{t}$ is the time reported in the legend. Note that all of them have been suitably rescaled for the sake of clarity, so they would not overlap with each other. Horizontal dotted black lines are additionally shown. Bottom panel: the same as in the upper panel but for the perpendicular velocity fluctuations, $\boldsymbol{u}_{\perp}$, compensated by $k_{\perp}^{3 / 2}$.

$\left[k_{0}, k^{\mathrm{inj}}\right]$, where $k_{0}$ is the largest scale corresponding to the computational box size, i.e., $k_{0}=2 \pi /\left(256 d_{\mathrm{p}}\right) \sim 0.025 d_{\mathrm{p}}^{-1}$. The nominal nonlinear time $\left.t_{\mathrm{NL}}\right|_{t=0}$ is different for each mode, being longer for lower $k$-vectors. As the system evolves, the injection scale gets larger and larger and most of the initial modes are involved in the development of the turbulent cascade at $t=200 \Omega_{\mathrm{p}}^{-1}$. Since modes with lower $k \mathrm{~s}$ keep feeding energy at large scales even afterwards, we expect turbulence to be still sustained also at later times. 


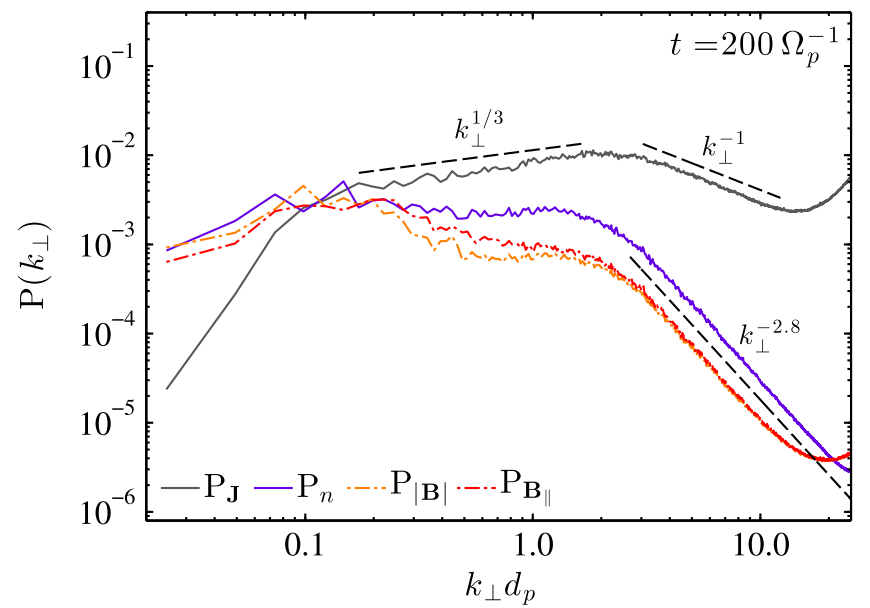

Figure 6. Spectra of the total current density, $\boldsymbol{J}$ (gray line), of the density fluctuations, $n$ (purple line), of the magnitude of the magnetic field, $|\boldsymbol{B}|$ (orange line), and of its parallel component, $\boldsymbol{B}_{\|}$(red dot-dashed line). Power laws with different spectral indices are additionally drawn in black dashed lines as a reference.

In Figure 5 we show the spectra of the perpendicular magnetic fluctuations, compensated by $k_{\perp}^{5 / 3}$ (top panel), and the spectra of the perpendicular velocity fluctuations, compensated by $k_{\perp}^{3 / 2}$ (bottom panel), computed at regular intervals of $50 \Omega_{\mathrm{p}}^{-1}$, from the maximum of turbulent activity to almost the end of the simulation. Here, the spectrum at a given time $\tilde{t}$ is the time-average between five different spectra corresponding to $\tilde{t}, \tilde{t} \pm 10 \Omega_{\mathrm{p}}^{-1}$ and $\tilde{t} \pm 20 \Omega_{\mathrm{p}}^{-1}$. The power-law scaling for both the magnetic and the velocity fluctuations are very well maintained, over about the same range, at all times $t>200 \Omega_{\mathrm{p}}^{-1}$, indicating that the turbulence decays in a selfsimilar way. Note that spectra corresponding to different times have been slightly shifted along the vertical axis in order to avoid overlapping.

In Figure 6, we show the spectra of the magnitude of the magnetic field, $|\boldsymbol{B}|$ (orange), of its parallel component, $\boldsymbol{B}_{\|}$(red dot-dashed), of the density fluctuations, $n$ (purple), and of the total current density, $\boldsymbol{J}$ (gray). The density and the parallel magnetic fluctuations are strongly coupled beyond $k_{\perp} d_{\mathrm{p}} \sim 2$. In the MHD range, they are approximately an order of magnitude smaller than the one of the perpendicular magnetic fluctuations. Therefore, the large-scale activity has little contribution from compressible fluctuations-although they can still play a significant role in the dynamics of the out-ofplane components - and the magnetic compressibility, i.e., the ratio of parallel to total magnetic fluctuations, is also negligible at small $k \mathrm{~s}$. Both spectra steepen at sub-proton scales, following a clean power-law scaling with a spectral index of -2.8 . Note that their relative power level with respect to other fields' spectra increases, with $P_{\boldsymbol{B}_{\|}}$(and also $P_{|\boldsymbol{B}|}$ ) becoming comparable with the spectrum of the perpendicular component, $\boldsymbol{B}_{\perp}$ (see Figure 4).

The spectral shape of the current density, $\boldsymbol{J}$, can be understood by recalling that $\boldsymbol{J}=\boldsymbol{\nabla} \times \boldsymbol{B}$. A simple order-ofmagnitude estimate of its perpendicular and parallel components gives $\boldsymbol{J}_{\perp} \propto k_{\perp} \boldsymbol{B}_{\|}$and $\boldsymbol{J}_{\|} \propto k_{\perp} \boldsymbol{B}_{\perp}$, respectively. Therefore, in the inertial range, where the magnetic activity is dominated by the perpendicular fluctuations, the spectrum of $\boldsymbol{J}$ is determined by its parallel component $\boldsymbol{J}_{\|}$and this results in the observed spectral index of $+1 / 3$, since $P_{\boldsymbol{J}_{\|}} \propto k_{\perp}^{2} P_{\boldsymbol{B}_{\perp}}$, with

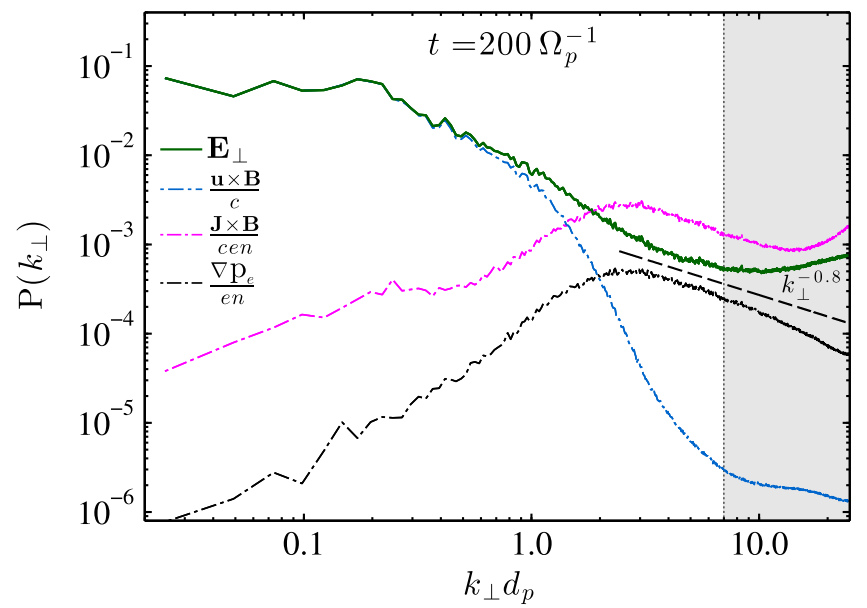

Figure 7. Spectrum of the perpendicular electric field, $\boldsymbol{E}_{\perp}$ (green solid line) and energy associated with the different terms of Equation (6) (the term containing the resistive coefficient is negligible). A power law with a spectral index of -0.8 is also drawn with a dashed black line as a reference. The shaded gray region marks the range where numerical effects strongly affect the shape of $P_{E_{\perp}}$.

$P_{\boldsymbol{B}} \propto k_{\perp}^{-5 / 3}$. On the contrary, as already discussed, $P_{\boldsymbol{B}_{\|}}$and $P_{\boldsymbol{B}}$ are comparable at sub-proton scales, both showing a power-law scaling, with spectral indices of -2.8 and -3 , respectively. The corresponding components, $\boldsymbol{J}_{\|}$and $\boldsymbol{J}_{\perp}$, are of the same order and exhibit a similar scaling, therefore $P_{\boldsymbol{J}} \sim P_{\boldsymbol{J}_{\|}} \sim P_{\boldsymbol{J}_{\perp}}$, and the corresponding scaling is in between $\propto k^{-0.8}$ and $\propto k^{-1}$. The change in the spectral slope of $P_{J}$ at $k_{\perp} d_{\mathrm{p}} \sim 2$ provides a further evidence of a spectral break in the magnetic field spectrum at proton scales.

Finally, the spectrum of the perpendicular electric fluctuations is reported in Figure 7 as a green line. We choose to pay particular attention to the electric field for three main reasons. Most importantly, it is expected to exhibit the most complex spectrum, since it contains the contributions of four terms having different relative importance in different ranges of scales. Second, it is the quantity that is mostly affected by both numerical effects and particle properties, so its behavior needs to be analyzed carefully, especially at small scales. Lastly, no consistent observational data about the properties of $\boldsymbol{E}$ are yet available, so making predictions about the shape of its spectrum can be relevant for future analysis. We recall here that starting from the Vlasov-fluid equations and assuming that the electrons act as a massless, charge-neutralizing fluid, the electric field can be computed from the generalized Ohm's law as

$$
\boldsymbol{E}=\underbrace{-\frac{\boldsymbol{u} \times \boldsymbol{B}}{c}}_{\boldsymbol{E}_{\mathrm{MHD}}}+\underbrace{\frac{\boldsymbol{J} \times \boldsymbol{B}}{c e n}}_{\boldsymbol{E}_{\mathrm{Hall}}} \underbrace{-\frac{\boldsymbol{\nabla} p_{\mathrm{e}}}{e n}}_{\boldsymbol{E}_{p_{\mathrm{e}}}}+\underbrace{\eta \boldsymbol{J}}_{\boldsymbol{E}_{\eta}} .
$$

In Figure 7, together with $P_{\boldsymbol{E}_{\downarrow}}$ obtained from numerical data, we also report the energy associated to the first three terms of Equation (6), computed a posteriori and drawn with cyan, magenta, and black dot-dashed lines, respectively (the contribution from the resistive term is negligible at all scales, since the resistive coefficient $\eta$ is $\left.5 \times 10^{-4}\right)$. At large scales, $P_{\boldsymbol{E}_{\perp}}$ is clearly dominated by the MHD term, $\boldsymbol{E}_{\mathrm{MHD}}$, which is essentially perpendicular to $\boldsymbol{B}_{0}$, since its leading contribution comes from $\boldsymbol{u}_{\perp} \times \boldsymbol{B}_{0}\left(\boldsymbol{u}_{\perp} \times \boldsymbol{B}_{\|}\right.$and $\boldsymbol{u}_{\|} \times \boldsymbol{B}_{\perp}$ are both of the 
second-order in the fluctuations). Therefore, $P_{\boldsymbol{E}_{\mid}}$strictly follows the spectrum of the perpendicular velocity fluctuations (see Figure 4). Approximately at $k_{\perp} d_{\mathrm{p}} \sim 0.5$, these two spectra decouple, since the second and third terms of Equation (6) start contributing.

We can accurately analyze the Hall term, $\boldsymbol{E}_{\mathrm{Hall}}$, by considering its perpendicular and parallel components separately. The former is of the first-order in the fluctuations, being led by $\left(\boldsymbol{k}_{\perp} \times \boldsymbol{B}_{\|}\right) \times \boldsymbol{B}_{0}$ (other contributions are quadratic in $\boldsymbol{B}_{\|}$ and $\boldsymbol{B}_{\perp}$, and therefore negligible). On the contrary, the latter is only of the second-order in the fluctuations, having the only contribution from $\left(\boldsymbol{k}_{\perp} \times \boldsymbol{B}_{\|}\right) \times \boldsymbol{B}_{\perp}$. Therefore, we expect the Hall term to be negligible at large scales, where $\boldsymbol{J}_{\perp}$ is small, and to exhibit a power-law behavior at small scales, with spectral index $\sim-0.8$ following from $E_{\text {Hall }} \propto k_{\perp}^{2} P_{\boldsymbol{B}_{\|}}$. Indeed, this is what we observe in Figure 7 (compare the magenta dot-dashed line with the reference dashed black line). The electron pressure gradient term, $\boldsymbol{E}_{p_{\mathrm{e}}}$, has only perpendicular components by construction (our 2D computational domain is perpendicular to $\boldsymbol{B}_{0}$ ). In the inertial range, it is of course negligible, the spectrum of the density fluctuations being essentially flat (compare with Figure 4). On the contrary, at small scales, it is expected to give a contribution $P_{\nabla n} \propto k_{\perp}^{2} P_{n}$, which has exactly the same slope as the contribution from the Hall term, since the spectra of the density fluctuations and of the parallel magnetic fluctuations have the same spectral index of -2.8 at sub-proton scales. This is indeed what we observe in Figure 7, where the contribution from the electron pressure gradient term is drawn with a black dot-dashed line. We would expect a similar behavior for $P_{\boldsymbol{E}_{\perp}}$ at sub-proton scales, i.e., a power law with a spectral index of $\sim-0.8$, and we observe a hint of a similar scaling in the range $2 \lesssim k_{\perp} d_{\mathrm{p}} \lesssim 7$.

The spectrum of the electric field fluctuations is the most affected by numerical effects among all the considered spectra, since the computation of $\boldsymbol{E}$ involves both other fields $(\boldsymbol{u}$ and $\boldsymbol{B})$ and derivatives $(\boldsymbol{\nabla} \times \boldsymbol{B}$ and $\boldsymbol{\nabla} n)$, as shown by Equation (6). We already noticed that $P_{\boldsymbol{B}}$ suffers from an accumulation of energy at small scales, which is only a numerical artifact, and so does $P_{\boldsymbol{J}}$ (see Figure 6). Moreover, derivatives in the numerical code are computed as finite differences, thus they are not able to recover very precise quantities at very small scales. For all these reasons, we cannot extract any robust information about the spectrum of the electric field at high wavevectors. In order to emphasize this, we choose to mark the "non-safety area," which we estimate as $k_{\perp} d_{\mathrm{p}} \gtrsim 7$, with a shaded gray region in Figure 7.

Under particular conditions, i.e., $T_{\mathrm{e}}=0$, one can obtain a better defined scaling for the electric field. In this case, the electron pressure gradient term, $\boldsymbol{E}_{p}$, in the Ohm's law is zero (see Equation (6)). Consequently, the level of the electric field spectrum at small scales is higher, since it is supported only by the Hall term $\boldsymbol{E}_{\mathrm{Hall}}$. This can be seen in Figure 8, where we show the same analysis of the electric field spectrum as in Figure 7, but for a case with $\beta_{\mathrm{e}}=0$ and all the other parameters set as in Run A. The electric field spectrum is now the sum of only two main contributions, $\boldsymbol{E}_{\mathrm{MHD}}$ and $\boldsymbol{E}_{\mathrm{Hall}}$. No qualitative changes are introduced with respect to the case with a finite electron temperature; the former term dominates the spectrum at MHD scales, while the latter is responsible for the flattening of the electric field at ion scales. The important difference with respect to Run A is that $P_{E_{1}}$ now displays a well-defined power-law slope with an index of -0.8 , consistent

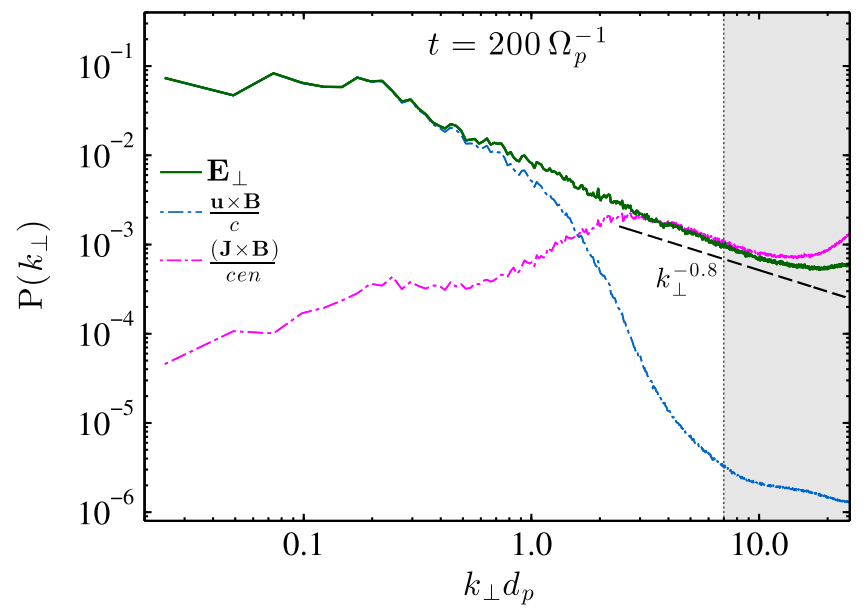

Figure 8. Spectrum of the perpendicular electric field, $\boldsymbol{E}_{\perp}$, as in Figure 7, for the case with $\beta_{\mathrm{e}}=0\left(\nabla p_{\mathrm{e}}=0\right)$.

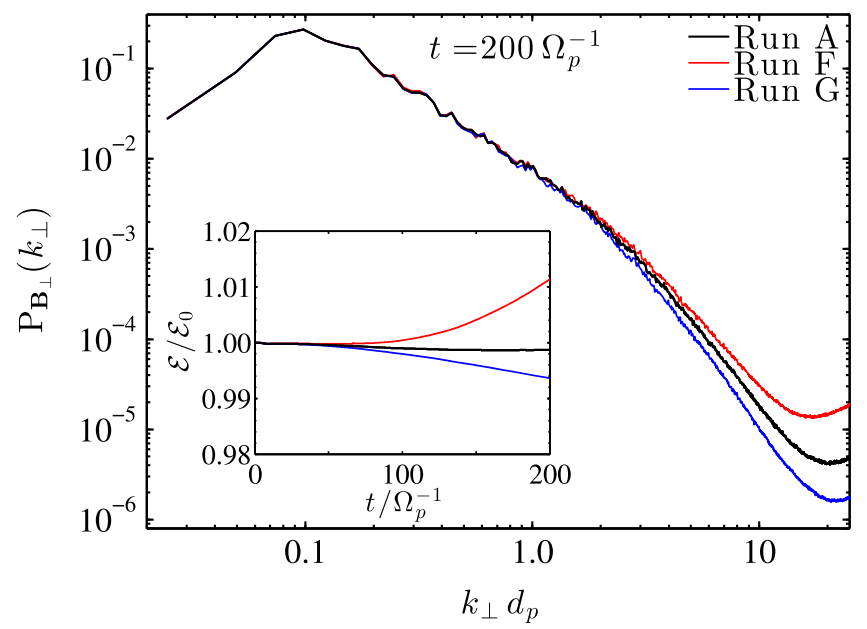

Figure 9. Power spectra of the perpendicular magnetic fluctuations, $\boldsymbol{B}_{\perp}$, for simulations with different values of the resistivity, i.e., Run A, $\left(\eta=5 \times 10^{-4}\right.$, black line), Run F $\left(\eta=1 \times 10^{-4}\right.$, red line), and Run $\mathrm{G}\left(\eta=1 \times 10^{-3}\right.$, blue line).

with the expectation. We expect the same slope also for the finite $T_{\mathrm{e}}$ case of Run $\mathrm{A}$ in the absence of the numerical limitations discussed above.

\section{ROLE OF NUMERICAL PARAMETERS}

\subsection{Spectral Properties}

As mentioned, a small numerical resistivity, $\eta$, has been implemented in all runs (see Table 1). A proper level of resistivity is mandatory in order to prevent an accumulation of the energy in magnetic fluctuations at small scales due to numerical errors. Runs $\mathrm{F}$ and $\mathrm{G}$ have been used to fine-tune the resistivity coefficient, starting from an order-of-magnitude estimate, and are characterized by the values $\eta=1 \times 10^{-4}$ and $1 \times 10^{-3}$, respectively. In Figure 9, we show the corresponding spectra of the perpendicular magnetic fluctuations, $\boldsymbol{B}_{\perp}$, at $t=200 \Omega_{\mathrm{p}}^{-1}$, in comparison with Run A $\left(\eta=5 \times 10^{-4}\right)$. For the setting adopted, the dissipative scale for the under-resistive case (Run F) can be estimated as $k_{\perp}^{\text {dis }} d_{\mathrm{p}} \sim 35$, i.e., smaller than the scale corresponding to the employed resolution. As a consequence, this value of the resistivity is not high enough to remove the energy excess at 
small scales, as also demonstrated by the shape of the spectrum of Run F in the sub-proton range. The over-resistive simulation (Run G) corresponds to the opposite case where $k_{\perp}^{\text {dis }} d_{\mathrm{p}} \sim 6$, thus well inside the range of wavevectors resolved in the simulation, making $P_{\boldsymbol{B}_{\boldsymbol{B}}}$ decreasing exponentially below the ion break, a clear indication of a too strong dissipative damping at sub-proton scales. Lastly, the intermediate case, i.e., Run A, is expected to introduce a dissipation scale $k_{\perp}^{\text {dis }} d_{\mathrm{p}} \sim 10$, allowing for the best description of the spectrum of $\boldsymbol{B}_{\perp}$. Indeed, this is observed to follow a power-law scaling with a spectral index of -3 for roughly a decade after the break (see Figure 4), in good agreement with solar wind spectra from observational data. Therefore, we decided to adopt $5 \times 10^{-4}$ as the optimal value for the resistive coefficient $\eta$.

As a further confirmation for the adequacy of our choice for $\eta$, in the insert of Figure 9 we also show the time evolution of the total energy $\mathcal{E}$, normalized to its initial value for the same three simulations. In all three cases, the total energy remains constant for $t \lesssim 70 \Omega_{\mathrm{p}}^{-1}$, while a different behavior is observed at later times. When the resistivity is too low (Run F, red line) $\mathcal{E}$ grows significantly due to the inefficient control of energy at small scales, and such an increase is already of the order of $\sim 1 \%$ at $t=200 \Omega_{\mathrm{p}}^{-1}$. On the contrary, when $\eta$ is too high (Run G, blue line) the action of resistivity is too strong. Note that part of the energy subtracted from the magnetic fluctuations would also go into electron heating, but, since the hybrid approximation does not provide an evolution for the electron temperature, such energy is not taken into account and is then lost by the system, resulting in a net decrease of $\mathcal{E}$. The value $\eta=5 \times 10^{-4}$ is the one which better ensures the conservation of the total energy, with a relative difference between the beginning and the end of the simulation, i.e., $t=500 \Omega_{\mathrm{p}}^{-1}$, of about $0.3 \%$. Also note that although the shape of $P_{\boldsymbol{B}}$ at sub-proton scales is quite strongly affected by the resistivity, the power-law scaling in the inertial range and the position of the spectral break are not, assuring the reliability of the spectra shown in Figures 4 and 5.

We also investigated the stability of omnidirectional spectra versus the spatial resolution. This was done by varying $\Delta x$, while keeping fixed the number of grid points (the total box length is then larger for larger grid spacing), as well as the amplitude of the initial magnetic fluctuations, $B^{\text {rms }}$. Runs $\mathrm{H}$ and I implement $\Delta x=0.250$ and $0.500 d_{\mathrm{p}}$, respectively (see Table 1). For both these runs, the value of the resistivity coefficient was suitably rescaled in order to get dissipation at the proper scales (note that $\eta \propto \Delta x$ under these conditions).

In Figure 10, the ratio between the omnidirectional spectra of the perpendicular electric and magnetic fluctuations, $P_{\boldsymbol{E}_{\perp}} / P_{\boldsymbol{B}_{\perp}}$, is compared among Runs A, H, and I. For all the three runs, this ratio exhibits the same scaling in the inertial range, following a power law with a spectral index of $1 / 6$. This is a direct consequence of the different scaling for the magnetic field $(-5 / 3)$ and the velocity $(-3 / 2)$ in the ideal MHD regimewhere also $P_{E_{\perp}} \sim P_{u_{\perp}}$-leading then to a spectral index of $-3 / 2+5 / 3=1 / 6$ for their ratio. As the other terms in the generalized Ohm's law became important at ion scales, the $P_{\boldsymbol{E}_{\perp}} / P_{\boldsymbol{B}_{\perp}}$ ratio increases significantly at smaller $k$ s. Interestingly, increasing $\Delta x$ from 0.125 (blue) to $0.250 d_{\mathrm{p}}$ (black) does not produce a change in the scale at which $P_{\boldsymbol{E}_{\perp}}$ exceeds $P_{\boldsymbol{B}_{\perp}}$, and this break is observed to occur at $k_{\perp} d_{\mathrm{p}} \sim 2$ in both cases. Moreover, the two curves exhibit similar slopes in the subproton ranges. This is a confirmation that the estimate of $\eta$ for

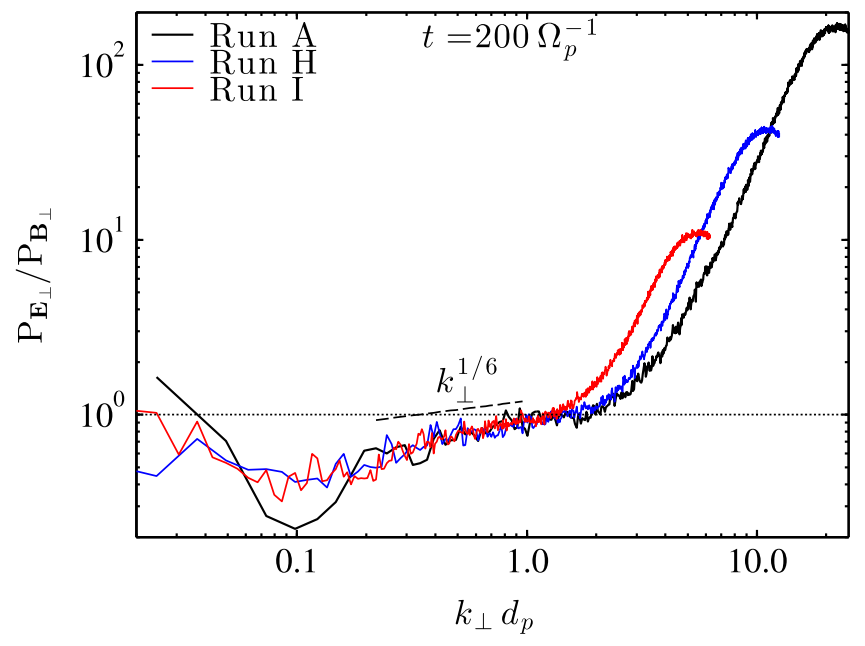

Figure 10. Ratio between the omnidirectional spectra of perpendicular electric fluctuations, $P_{\boldsymbol{E}_{1}}$, and perpendicular magnetic fluctuations, $P_{\boldsymbol{B}}$, for simulations with different resolution: Run A, with $\Delta x=0.125 d_{\mathrm{p}}$ (black line), Run $\mathrm{H}$, with $\Delta x=0.25 d_{\mathrm{p}}$ (blue line), and Run I, with $\Delta x=0.5 d_{\mathrm{p}}$ (red line).

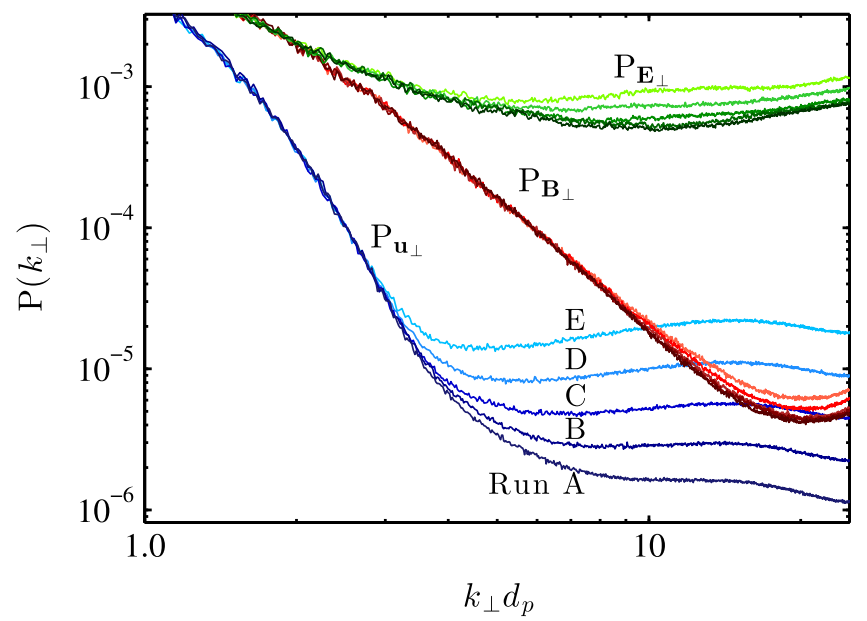

Figure 11. Zoom of the power spectra of the perpendicular magnetic, electric, and proton bulk velocity fluctuations (drawn with red, green, and blue lines, respectively) at small scales, where the contribution of numerical noise is not negligible. Lines with different shades of the same color correspond to simulations with different amounts of ppc, ranging from 500 to 8000 , with darker colors being associated with a higher number of particles.

the two simulations was correct and that the raise in $P_{\boldsymbol{E}_{1}} / P_{\boldsymbol{B}_{\boldsymbol{B}}}$ is physical and well captured by the runs. On the other hand, when employing a lower resolution, $\Delta x=0.500$ (red), the break seems to occur at slightly larger scales. This is likely a consequence of the reduction of the resolution at small scales: in Run I, the break and the dissipative scale are not well separated in Fourier space, so that subtracting energy at the smallest scales via resistive dissipation also affects the shape of the spectra around $k_{\perp} d_{\mathrm{p}} \sim 2$, where the break occurs. This is evidence that the scaling for the spectra discussed and shown in Figure 4 continue to hold at lower spatial resolution, but also that $\Delta x \gtrsim 0.500 d_{\mathrm{p}}$ is not sufficient to properly explore the physical behavior at sub-ion scales.

Finally, the importance of employing a large number of particles was investigated by keeping all the same parameters as for Run A except for varying the number of ppc from 4000 to 500 in steps of a factor of 2 (Runs from B to E in Table 1). In Figure 11, the spectra of the perpendicular velocity, 


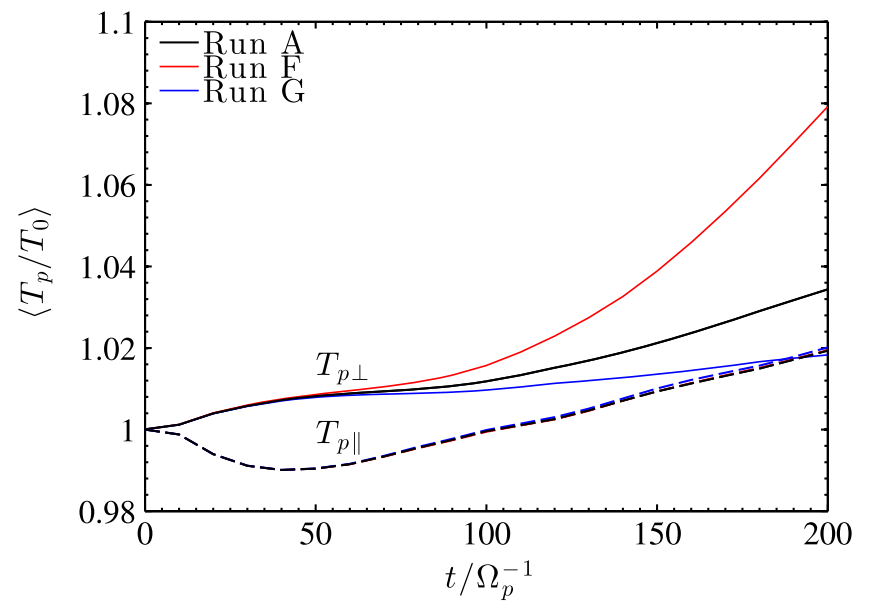

Figure 12. Time evolution of the parallel and perpendicular proton temperature, $T_{\mathrm{p} \|}$ and $T_{\mathrm{p} \perp}$, respectively, normalized to the initial common value, $T_{0}$. The evolution is here shown for different values of the resistive coefficient (see Table 1). Solid and dashed lines are used for $T_{\mathrm{p} \perp}$ and $T_{\mathrm{p} \|}$, respectively.

magnetic, and electric fluctuations are reported with lines in different shades of blue, red, and green, respectively, corresponding to simulations with different numbers of ppc ranging from 500 (lighter color, Run E) to 8000 (darker color, Run A). Increasing the number of pcc from $500\left(\sim 2.0 \times 10^{9}\right.$ total particles $)$ to $8000\left(\sim 3.3 \times 10^{10}\right.$ total particles $)$ results in a decrease of the noise at small scales of more than one order of magnitude for the spectrum of the perpendicular velocity fluctuations. On the other hand, the trend of the spectrum up to the proton inertial length and slightly above is not affected, and the curve corresponding to our most accurate simulation (Run A) overlaps with the one with $4000 \mathrm{ppc}$ (Run B) up to $k_{\perp} d_{\mathrm{p}} \sim 4$. On the contrary, the spectrum of the perpendicular magnetic fluctuations is only barely affected by the numerical noise when the number of particles is sufficiently high, since the curves for 4000 and 8000 ppc are almost indistinguishable, proving that the number of ppc employed in Run A is sufficient to obtain reliable results for $P_{\boldsymbol{B}_{\perp}}$ up to $k_{\perp} d_{\mathrm{p}} \sim 10$. Lastly, the spectrum of the perpendicular electric fluctuations shows a dependence on the number of particles at scales $k_{\perp} d_{\mathrm{p}} \gtrsim 4$, but the curves for 4000 and $8000 \mathrm{ppc}$ are quite close to each other even at smaller scales. However, as mentioned, $P_{\boldsymbol{E}}$ is influenced by different sources of numerical noise, and all contribute in affecting the spectrum at small scales. We stress that the evaluation of the noise due to the finite number of ppc only represents a lower limit of the overall noise, and therefore our previous choice of marking a shaded gray area for $k_{\perp} d_{\mathrm{p}} \gtrsim 7$ in Figure 7 is not in contrast with these results.

\subsection{Proton Heating}

Figure 1 shows that some particle heating is observed during the turbulent activity. Some care must be used in the interpretations of this result, since it may be significantly affected by some of the numerical settings. Therefore, we carefully consider the properties of the proton heating in this subsection.

Resistivity is observed to play a fundamental role in determining the overall proton heating, $\Delta T_{\|, \perp}=\left\langle T_{\|, \perp}\right\rangle-T_{0}$, and the proton temperature anisotropy, $A_{\mathrm{p}}$. In Figure 12, we show the time evolution of the perpendicular $T_{\mathrm{p} \perp}$ and the

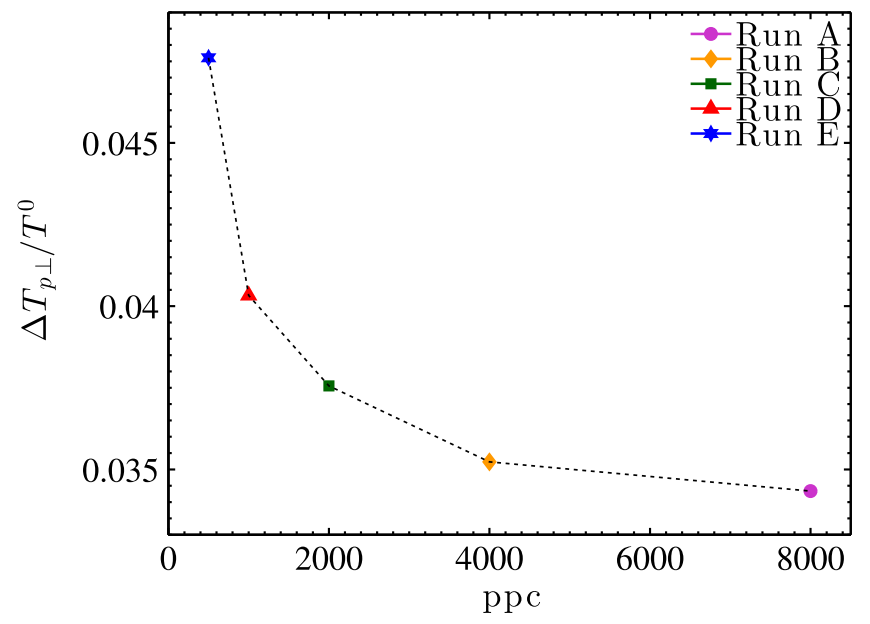

Figure 13. Perpendicular proton heating, $\Delta T_{\mathrm{p} \perp} / T_{0}$, computed at $t=200 \Omega_{\mathrm{p}}^{-1}$, vs. the number of ppc employed, ranging from 8000 (Run A) to 500 (Run E).

parallel $T_{\mathrm{p} \|}$ proton temperature, in solid and dashed lines, respectively, corresponding to different values of the resistive coefficient $\eta$ (Runs A, F, and G of Table 1). The time evolution of $T_{\mathrm{p} \|}$ is observed to be almost unaffected by the resistivity, showing an early decrease up to $t \sim 50 \Omega_{\mathrm{p}}^{-1}$ and then an increase with an almost constant rate, as was already shown for Run A in Figure 1. The situation is different for $T_{\mathrm{p} \perp}$, since its behavior for different values of $\eta$ is the same only during the initial readjustment of the system, while it starts to differ after $t \sim 40 \Omega_{\mathrm{p}}^{-1}$. At later times, $T_{\mathrm{p} \perp}$ exhibits a growth rate very similar to that of $T_{\mathrm{p} \|}$ for Run A (black), so no preferential perpendicular or parallel heating is observed. In particular, $\Delta T_{\mathrm{p} \perp} / T_{0}$ at $t=200 \Omega_{\mathrm{p}}^{-1}$ is about $3.5 \%$, while the corresponding $\Delta T_{\mathrm{p} \|} / T_{0}$ is about $2 \%$. When $\eta$ is lower (Run F, red), $T_{\mathrm{p} \perp}$ grows at a much faster rate than $T_{\mathrm{p} \|}$ for $t \gtrsim 50 \Omega_{\mathrm{p}}^{-1}$, generating a strong preferential heating in the perpendicular direction, $T_{\mathrm{p} \perp}$ being about $8 \%$ greater than the initial value at $t=200 \Omega_{\mathrm{p}}^{-1}$. On the contrary, when $\eta$ is higher (Run G, black lines), $T_{\mathrm{p} \perp}$ grows much more slowly, and is overcome by $T_{\mathrm{p} \|}$ just before $t=200 \Omega_{\mathrm{p}}^{-1}$, leading to $A_{\mathrm{p}}<1$ at later times. The amount of perpendicular heating observed is then significantly related to the presence of an excess of fluctuations at small scales, and can therefore then be largely overestimated or underestimated if an incorrect value of the resistivity is adopted.

The perpendicular proton heating is also found to be strongly affected by the number of particles employed. In Figure 13, we report the ratio $\Delta T_{\mathrm{p} \perp} / T_{0}$, where $\Delta T_{\mathrm{p} \perp}=\left\langle T_{\mathrm{p} \perp}\right\rangle-T_{0}$ is computed at $t=200 \Omega_{\mathrm{p}}^{-1}$, versus the number of ppc for Runs A-E (see Table 1). At this time, $\Delta T_{\mathrm{p} \perp} / T_{0}$ is clearly higher when employing a lower number of ppc. In particular, while a good convergence toward a value of $\sim 3.4 \%$ is observed when increasing the number of ppc from 4000 to 8000, this quantity clearly diverges when few particles are employed, reaching $4.8 \%$ for $500 \mathrm{ppc}$. Moreover, the difference in $T_{\mathrm{p} \perp}$ between Run A and Run E tends to further increase at later times. The main result of this analysis is that the use of a large number of ppc is mandatory when trying to give an estimate of $T_{\mathrm{p} \perp}$, which could be largely overestimated otherwise. On the contrary, the parallel proton temperature is found to be largely independent from the number of particles (the relative difference between Run $\mathrm{A}$ and Run $\mathrm{E}$ is lower than $0.1 \%$ at $t=200 \Omega_{\mathrm{p}}^{-1}$ ). 
To conclude our analysis, we find that the spatial resolution $\Delta x$ does not seem to significantly affect the proton heating, provided that the value of the resistivity is suitably set as discussed in Section 4.1. Differences between $T_{\mathrm{p} \perp}$ at $t=200 \Omega_{\mathrm{p}}^{-1}$ for Runs $\mathrm{A}, \mathrm{H}$, and I are less than $0.6 \%$. The dependency of $T_{\mathrm{p} \|}$ on the spatial resolution is also negligible.

\section{SUMMARY AND CONCLUSIONS}

In this work, we have presented properties of turbulence in a magnetized collisionless plasma by means of 2D hybrid PIC simulations, extending the results of Letter 1. Remarkably, our simulations implement a high number of collocation points $(2048 \times 2048)$ and a very high number of particles (up to 8000 ppc), covering a large simulation domain (not less than $L_{\text {box }}=256 d_{\mathrm{p}}$ ) with a fine spatial resolution. This enables us to self-consistently describe the evolution of turbulence over three orders of magnitude in wavevectors, and to fully capture its transition from fluid-like MHD scales to kinetic sub-ion scales, by using a single simulation (see Letter 1).

The adopted initial conditions consist of balanced and equipartitioned magnetic and velocity fluctuations, i.e., with zero cross helicity and zero residual energy. The onset of a turbulent cascade appears quite early during the simulations $\left(t \sim 200 \Omega_{\mathrm{p}}^{-1}\right.$, corresponding to approximatively 10 nonlinear times $t_{\mathrm{NL}}$ ), i.e., when most of the initial modes have started to partake into the cascade. In physical space, the activity of turbulence is characterized by magnetic field coherent structures, vortices, and strong and localized current sheets at smaller scales.

The generation of coherent structures associated to intermittency is observed as turbulence evolves through MHD to sub-proton scales. PDFs of increments of a perpendicular component of the magnetic fluctuations at $t=200 \Omega_{\mathrm{p}}^{-1}$ exhibit a deviation from the normal distribution at all scales. This is small in the inertial range, becoming larger in correspondence of the spectral break at $k_{\perp} d_{\mathrm{p}} \sim 2$, while at $k_{\perp} d_{\mathrm{p}}>2$ the PDF has a much leaner shape with long non-zero tails. The corresponding excess kurtosis confirms this behavior. It is very small around the injection scale, since part of the MHD range fluctuations still acts as an energy reservoir for turbulence at $t=200 \Omega_{\mathrm{p}}^{-1}$, while it increases through the inertial range. Moreover, we observe a further increase at smaller scales. Observational data give no firm results about the behavior of this quantity at different scales (Alexandrova et al. 2008a; Kiyani et al. 2009, 2013; Wu et al. 2013; Chen et al. 2014). Nevertheless, when shown, all previous simulations observe an increase of the kourtosis at smaller scales (e.g., Dmitruk \& Matthaeus 2006; Wan et al. 2012; Karimabadi et al. 2013; Wu et al. 2013).

When looking at the spectra of the relevant quantities, two clear distinct turbulent regimes are observed. At larger scales, the magnetic field follows a Kolmogorov $-5 / 3$ power law, while the velocity has a spectral index of $-3 / 2$, which is characteristic of a Iroshnikov-Kraichnan turbulence. An excess of magnetic energy with respect to the kinetic energy is observed throughout the inertial range. The two different scalings for the magnetic and velocity fluctuations, often observed in the solar wind (Podesta et al. 2006, 2007; Salem et al. 2009; Tessein et al. 2009; Chen et al. 2011b), are very stable in time. They appear at the maximum of the turbulent activity and persist throughout all the simulations, as the energy reservoir at large scales is able to sustain and maintain the cascade. In Letter 1, we showed that such magnetic and velocity scaling are also combined with a spectral index of -2 for the residual energy, in agreement with observations in the solar wind (Chen et al. 2013a). Incompressible MHD (Müller \& Grappin 2005) and RMHD (Boldyrev et al. 2011) only partially reproduce such scaling. In our simulations, the 2D geometry and the presence of compressibility may play a role in setting the different scaling (see also Grappin et al. 2015 for similar findings in compressible 3D MHD decaying turbulence).

A clear transition in the spectra is observed at scales $k_{\perp} d_{\mathrm{p}} \gtrsim 1$, with a change in the spectral indices of all fields. In particular, the spectrum of the perpendicular magnetic fluctuations steepens at $k_{\perp} d_{\mathrm{p}} \sim 2$, following a power law with a spectral index $\sim-3$ for another decade. The location of the break does not show any significant dependence on the number of particles, the spatial resolution, and the resistivity adopted, provided that a sufficient number of grid points allows us to cover approximatively a decade at sub-proton scales, i.e., that the scale at which resistive dissipation acts is sufficiently separated from the region of the break. The parallel component of the magnetic field, together with the density, follows a similar but slightly shallower slope with a spectral index of $\sim-2.8$, in very good agreement with observations (Chen et al. 2012, 2013b) and other simulations (Howes et al. 2011; Passot et al. 2014). As a result, magnetic fluctuations tend to become isotropic at small scales, resulting in an increase of the magnetic compressibility, as observed in the solar wind (Podesta \& TenBarge 2012; Salem et al. 2012; Kiyani et al. 2013). The spectrum of the perpendicular velocity fluctuations quickly drops above $k_{\perp} d_{\mathrm{p}} \sim 1$, without any clear power-law trend. The observation of a spectral index of -2.8 has been ascribed to the effect of the electron Landau damping by previous studies (Howes et al. 2011; Passot et al. 2014); however, this cannot be the case in our simulations, where the electron kinetics is not taken into account. Alternatively, the presence of coherent structures, such as current sheets, can produce a steepening of the energy spectra (e.g., Wan et al. 2012; Karimabadi et al. 2013). The increase of intermittency at small scales, observed in our simulations, seems to confirm this path toward the dissipation. We have to note, however, that a $-8 / 3$ power law for the magnetic energy and the density spectra (not far from the 2.8 found here) has been also interpreted as related to the dimensionality (1D or 2D) of the magnetic and the density intermittent structures, without invoking dissipation (Boldyrev \& Perez 2012; Meyrand \& Galtier 2013).

The spectrum of the electric fluctuations is highly dominated by its perpendicular component. It is strongly coupled to the spectrum of the perpendicular velocity fluctuations at fluid scales, then it decouples and flattens, exceeding the spectrum of the perpendicular magnetic fluctuations and becoming dominant for $k_{\perp} d_{\mathrm{p}} \gtrsim 2$. At large scales, the only contribution comes from the MHD term in Equation (6), whose leading term is

$$
\boldsymbol{E}_{\perp} \propto \boldsymbol{u} \times \boldsymbol{B} \sim \boldsymbol{u}_{\perp} \times \boldsymbol{B}_{0} .
$$

This corresponds to a power-law scaling

$$
P_{\boldsymbol{E}} \propto P_{\boldsymbol{u}_{\perp}} \propto k_{\perp}^{-3 / 2},
$$

which is observed in the simulations and is also consistent with observations (Chen et al. 2011a). In our case, the main 
contributions at sub-proton scales come from the Hall and the electron pressure gradient terms, since the spectrum of the velocity fluctuations is observed to drop exponentially at short wavelengths. The leading terms at these scales are then

$$
\boldsymbol{J} \times \boldsymbol{B}-\nabla p_{\mathrm{e}} \propto \nabla\left(T_{\mathrm{e}} n+\boldsymbol{B}_{0} \cdot \boldsymbol{B}_{\|}\right) .
$$

Note that in general, the sum of the two terms inside parentheses would not necessarily result in a power law for the electric field. However, since in our simulations both $n$ and $\boldsymbol{B}_{\|}$are observed to scale with the same power law-due to the strong coupling between the plasma and the magnetic compressibility - then the expected spectral index for the electric field is

$$
P_{\boldsymbol{E}} \propto k_{\perp}^{2} P_{\boldsymbol{B}_{\|, n}} \propto k_{\perp}^{-0.8}
$$

Although it is not possible to directly test this scaling for the electric field spectrum in Run A, individual terms in Equation (10) follow well the prediction (see Figure 7). Moreover, we were able to show that when assuming $T_{\mathrm{e}}=0$ (i.e., setting to zero the electron pressure gradient term in the Ohm's law), then the electric field spectrum-hence dominated by the Hall term-follows a $k_{\perp}^{-0.8}$ scaling in the sub-proton range (Figure 8), and is only very slightly affected by numerical effects at very small scales $\left(k_{\perp} d_{\mathrm{p}} \gtrsim 10\right)$.

As a result of the interaction of particles with the turbulent fluctuations and small-scale structures, we observe an overall parallel and perpendicular heating with similar rates, so that the temperature of the plasma remains globally nearly isotropic. This behavior can be achieved only if a large enough number of particles is employed, and the resistivity is properly set in order to assure an accurate conservation of the total energy and a clear power-law behavior for the spectrum of the magnetic fluctuations at all scales. The parallel temperature, $T_{\mathrm{p} \|}$, is found to have a very robust evolution, being essentially independent of the resistivity, the number of particles, and the spatial resolution employed. On the contrary, the time evolution of $T_{\mathrm{p} \perp}$ is strongly determined by both the resistivity and the number of ppc: if too few particles are employed, or if the resistivity is too low, the perpendicular heating can be largely overestimated/unphysical. Conversely, when a too strong value of the resistivity is implemented, the artificial damping of fluctuations at ion scales can produce a strong reduction of the perpendicular heating, thus generating an equally unphysical preferential parallel heating. This proves that no firm conclusions can be drawn about the perpendicular heating by turbulence in hybrid simulations, unless a careful and empirically fine-tuned choice of all parameters has been taken.

Note, however, that the fact that we do not observe a global preferential heating does not imply the absence of signatures of localized preferential deformations of the particle distribution functions, as suggested by the bottom right panel of Figure 2, where strong temperature anisotropies ranging from 0.5 to 1.8 are observed. They seem to be concentrated in regions with stronger coherent structures, identified by the presence of current sheets and a significant level of vorticity. These results are in agreement with previous works based on the Vlasovhybrid approximation (e.g., Servidio et al. 2012; Perrone et al. 2013; Servidio et al. 2014; Valentini et al. 2014). As the overall heating is rather weak, slow, and nearly isotropic, we can infer that the local formation of large proton temperature anisotropies is likely due to energy exchanges between the parallel and perpendicular directions, and/or to the spatial transport, rather than due to the heating.

Solar wind observations show a certain variability of the spectral properties. In particular, the position of the break at ion scales and the shape of the magnetic field spectrum around it seems to depend on the power of magnetic fluctuations (Bruno et al. 2014) and on the plasma beta (Alexandrova et al. 2008a; Chandran et al. 2009; Chen et al. 2014). Investigating such a dependence, by exploring the parameter space, will be the subject of a fortcoming paper.

The 2D geometry strongly constrains the simulation results of turbulence, since this process is inherently 3D. In particular, a 2D geometry affects significantly the propagation of modes, inhibits the presence of small-scale parallel waves, constrains the role of possible competing mechanisms, such as MHD (e.g., Landi et al. 2008; Landi \& Bettarini 2012) and kinetic (e.g., Hellinger et al. 2015) instabilities, and limits the shape and size of dissipative structures (Wan et al. 2012, 2015). On the other hand, recent 3D studies confirmed some of the properties observed in 2D simulations, e.g., the overall heating (Vasquez et al. 2014; Vasquez 2015; Wan et al. 2015), the local formation of complex ion distribution functions (Servidio et al. 2015), and the impact of microphysics on current sheets stability (Gingell et al. 2015). However, spectral properties are not yet well resolved in three dimensions since, in order to infer a reliable spectral behavior over a wide range of scales, as well as to quantitatively estimate the proton heating, a very high accuracy is mandatory (i.e., a very high spatial resolution and a very large number of particles for PIC models, or a very high phase space resolution for Vlasov-Maxwell models). Therefore, high-resolution 2D studies can still represent an optimal and meaningful compromise in all thoses cases which would be prohibitive at present in three dimensions, e.g., when exploring a parameter space (Parashar et al. 2015), or when performing very long expanding-box simulations (Hellinger et al. 2015). Moreover, in our configuration, the most important component of the MHD cascade, i.e., Alfvén modes, are clearly present, since the ambient magnetic field is out-of-plane and the initial fluctuations are perpendicular to both the wave vector and the mean field. The propagation of Alfvén modes along the direction of the global mean field is inhibited by construction. However, they can propagate with finite, yet low, frequencies with respect to the local mean field, the fluctuations being relatively strong $\left(B^{\mathrm{rms}} / B_{0} \sim 1 / 4\right)$. For these reasons, the limitations of our $2 \mathrm{D}$ simulations, although present, are not as strong or unrealistic as they could seem at first glance. The very good agreement between our results and solar wind observations, although a priori unexpected, may represent itself a sort of a posteriori evalutation of the soundness of our 2D study. Of course, all quantitative results from the present work, as well as from previous 2D studies in the literature, need to be verified and validate by realistic $3 \mathrm{D}$ simulations. 3D simulations, employing a similar setting and similar choices for the main parameters as the ones adopted in the present paper, will be the subject of future work.

The authors acknowledge valuable discussions with Olga Alexandrova, Chris Chen, Giuseppe Consolini, Roland Grappin, Frank Löffler, and Marco Velli.

This project has received funding from the European Unions Seventh Framework Programme for research, technological 
development and demonstration under grant agreement No. 284515 (SHOCK). Website: http://project-shock.eu/home/.

This research was conducted with high performance computing (HPC) resources provided by the Louisiana State University (allocations hpc_hyrel14 and hpc_hyrel15) and by CINECA (grant HP10CVCUF1).

P.H. acknowledges the GACR grant 15-10057S. L.M. was funded by STFC grant ST/K001051/1. A.V. acknowledges the Interuniversity Attraction Poles Programme initiated by the Belgian Science Policy Office (IAP P7/08 CHARM).

\section{REFERENCES}

Alexandrova, O., Carbone, V., Veltri, P., \& Sorriso-Valvo, L. 2008a, ApJ, 674,1153

Alexandrova, O., Chen, C. H. K., Sorriso-Valvo, L., Horbury, T. S., \& Bale, S. D. 2013, SSRv, 178, 101

Alexandrova, O., Lacombe, C., \& Mangeney, A. 2008b, AnGeo, 26, 3585

Alexandrova, O., Lacombe, C., Mangeney, A., Grappin, R., \& Maksimovic, M. 2012, ApJ, 760, 121

Alexandrova, O., Saur, J., Lacombe, C., et al. 2009, PhRvL, 103, 165003

Bale, S. D., Kellogg, P. J., Mozer, F. S., Horbury, T. S., \& Reme, H. 2005, PhRvL, 94, 215002

Beinroth, H. J., \& Neubauer, F. M. 1981, JGR, 86, 7755

Beresnyak, A. 2011, PhRvL, 106, 075001

Beresnyak, A., \& Lazarian, A. 2009, ApJ, 702, 460

Biskamp, D., Schwarz, E., Zeiler, A., Celani, A., \& Drake, J. F. 1999, PhPl, 6,751

Boldyrev, S., \& Perez, J. C. 2012, ApJL, 758, L44

Boldyrev, S., Perez, J. C., Borovsky, J. E., \& Podesta, J. J. 2011, ApJL, 741, L19

Bruno, R., \& Carbone, V. 2013, LRSP, 10, 2

Bruno, R., Trenchi, L., \& Telloni, D. 2014, ApJL, 793, L15

Camporeale, E., \& Burgess, D. 2011, ApJ, 730, 114

Chandran, B. D. G., Quataert, E., Howes, G. G., Xia, Q., \& Pongkitiwanichakul, P. 2009, ApJ, 707, 1668

Chang, O., Gary, P. S., \& Wang, J. 2011, GeoRL, 38, L22102

Chen, C. H. K., Bale, S. D., Salem, C., \& Mozer, F. S. 2011a, ApJL, 737, L41

Chen, C. H. K., Bale, S. D., Salem, C. S., \& Maruca, B. A. 2013a, ApJ, 770,125

Chen, C. H. K., Boldyrev, S., Xia, Q., \& Perez, J. C. 2013b, PhRvL, 110, 225002

Chen, C. H. K., Horbury, T. S., Schekochihin, A. A., et al. 2010, PhRvL, 104, 255002

Chen, C. H. K., Leung, L., Boldyrev, S., Maruca, B. A., \& Bale, S. D. 2014, GeoRL, 41, 8081

Chen, C. H. K., Mallet, A., Yousef, T. A., Schekochihin, A. A., \& Horbury, T. S. 2011 b, MNRAS, 415, 3219

Chen, C. H. K., Salem, C. S., Bonnell, J. W., Mozer, F. S., \& Bale, S. D. 2012, PhRvL, 109, 035001

Chen, C. H. K., Sorriso-Valvo, L., Šafránková, J., \& Němeček, Z. 2014, ApJL, 789, L8

Cho, J., \& Lazarian, A. 2004, ApJL, 615, L41

Cho, J., \& Lazarian, A. 2009, ApJ, 701, 236

Cranmer, S. R., Matthaeus, W. H., Breech, B. A., \& Kasper, J. C. 2009, ApJ, 702, 1604

Dmitruk, P., \& Matthaeus, W. H. 2006, PhPl, 13, 042307

Dong, Y., Verdini, A., \& Grappin, R. 2014, ApJ, 793, 118

Franci, L., Verdini, A., Matteini, L., Landi, S., \& Hellinger, P. 2015, ApJL, 804, L39

Gingell, P. W., Burgess, D., \& Matteini, L. 2015, ApJ, 802, 4

Goldstein, M. L., Roberts, D. A., \& Fitch, C. A. 1994, JGR, 99, 11519

Gómez, D., Martín, L. N., \& Dmitruk, P. 2013, AdSpR, 51, 1916

Gómez, D. O., Mahajan, S. M., \& Dmitruk, P. 2008, PhPl, 15, 102303

Grappin, R., Mangeney, A., \& Marsch, E. 1990, JGR, 95, 8197

Grappin, R., \& Müller, W.-C. 2010, PhRvE, 82, 026406

Grappin, R., Müller, W.-C., \& Verdini, A. 2015, ApJL, submitted

Grappin, R., Velli, M., \& Mangeney, A. 1991, AnGeo, 9, 416

Hellinger, P., Matteini, L., Landi, S., et al. 2015, ApJL, 811, L32

Hellinger, P., Matteini, L., Štverák, Š, Trávníček, P. M., \& Marsch, E. 2011, JGR, 116, A09105

Hellinger, P., Trávníček, P., Kasper, J. C., \& Lazarus, A. J. 2006, GeoRL, 33, L09101
Hellinger, P., Trávníček, P. M., Štverák, Š, Matteini, L., \& Velli, M. 2013, JGR, 118, 1351

Howes, G. G., Dorland, W., Cowley, S. C., et al. 2008, PhRvL, 100, 065004 Howes, G. G., Tenbarge, J. M., Dorland, W., et al. 2011, PhRvL, 107, 035004

Karimabadi, H., Roytershteyn, V., Wan, M., et al. 2013, PhPl, 20, 012303

Kiyani, K. H., Chapman, S. C., Khotyaintsev, Y. V., Dunlop, M. W., \& Sahraoui, F. 2009, PhRvL, 103, 075006

Kiyani, K. H., Chapman, S. C., Sahraoui, F., et al. 2013, ApJ, 763, 10

Landi, S., \& Bettarini, L. 2012, SSRv, 172, 253

Landi, S., Londrillo, P., Velli, M., \& Bettarini, L. 2008, PhPl, 15, 012302

Leamon, R. J., Smith, C. W., Ness, N. F., Matthaeus, W. H., \& Wong, H. K. 1998, JGR, 103, 4775

Leamon, R. J., Smith, C. W., Ness, N. F., \& Wong, H. K. 1999, JGR, 104, 22331

Lee, E., Brachet, M. E., Pouquet, A., Mininni, P. D., \& Rosenberg, D. 2010 , PhRvE, 81, 016318

MacBride, B. T., Smith, C. W., \& Forman, M. A. 2008, ApJ, 679, 1644

Markovskii, S. A., \& Vasquez, B. J. 2011, ApJ, 739, 22

Markovskii, S. A., Vasquez, B. J., \& Chandran, B. D. G. 2010, ApJ, 709, 1003

Maron, J., \& Goldreich, P. 2001, ApJ, 554, 1175

Marsch, E., Ao, X.-Z., \& Tu, C.-Y. 2004, JGR, 109, 4102

Marsch, E., Schwenn, R., Rosenbauer, H., et al. 1982, JGR, 87, 52

Martin, L. N., De Vita, G., Sorriso-Valvo, L., et al. 2013, PhRvE, 88, 063107

Mason, J., Cattaneo, F., \& Boldyrev, S. 2008, PhRvE, 77, 036403

Matteini, L., Hellinger, P., Goldstein, B. E., et al. 2013a, JGRA, 118, 2771

Matteini, L., Landi, S., Hellinger, P., et al. 2007, GeoRL, 34, 20105

Matteini, L., Landi, S., Velli, M., \& Matthaeus, W. H. 2013b, ApJ, 763, 142

Matthaeus, W. H., Dmitruk, P., Smith, D., Ghosh, S., \& Oughton, S. 2003, GeoRL, 30, 2104

Matthaeus, W. H., \& Velli, M. 2011, SSRv, 160, 145

Matthews, A. P. 1994, JCoPh, 112, 102

Meyrand, R., \& Galtier, S. 2013, PhRvL, 111, 264501

Mininni, P. D., \& Pouquet, A. 2007, PhRvE, 99, 1

Mininni, P. D., \& Pouquet, A. 2009, PhRvE, 80, 025401

Müller, W.-C., Biskamp, D., \& Grappin, R. 2003, PhRvE, 67, 066302

Müller, W.-C., \& Grappin, R. 2005, PhRvL, 95, 114502

Ng, C. S., Bhattacharjee, A., Germaschewski, K., \& Galtier, S. 2003, PhPl, 10,1954

Parashar, T. N., Salem, C., Wicks, R. T., et al. 2015, JPlPh, 81, 905810513

Parashar, T. N., Servidio, S., Breech, B., Shay, M. A., \& Matthaeus, W. H 2010, PhPl, 17, 102304

Parashar, T. N., Shay, M. A., Cassak, P. A., \& Matthaeus, W. H. 2009, PhPl, 16,032310

Parashar, T. N., Vasquez, B. J., \& Markovskii, S. A. 2014, PhPl, 21, 022301

Passot, T., Henri, P., Laveder, D., \& Sulem, P.-L. 2014, EPJD, 68, 207

Perez, J. C., \& Boldyrev, S. 2009, PhRvL, 102, 025003

Perez, J. C., Mason, J., Boldyrev, S., \& Cattaneo, F. 2012, PhRvX, 2, 041005

Perrone, D., Valentini, F., Servidio, S., Dalena, S., \& Veltri, P. 2013, ApJ, 762, 99

Perrone, D., Valentini, F., Servidio, S., Dalena, S., \& Veltri, P. 2014, EPJD, 68, 209

Podesta, J. J., \& Borovsky, J. E. 2010, PhPl, 17, 112905

Podesta, J. J., Roberts, D. A., \& Goldstein, M. L. 2006, JGR, 111, A10109

Podesta, J. J., Roberts, D. A., \& Goldstein, M. L. 2007, ApJ, 664, 543

Podesta, J. J., \& TenBarge, J. M. 2012, JGR, 117, A10106

Rodriguez Imazio, P., Martin, L. N., Dmitruk, P., \& Mininni, P. D. 2013, PhP1, 20, 052506

Sahraoui, F., Goldstein, M. L., Belmont, G., Canu, P., \& Rezeau, L. 2010, PhRvL, 105, 131101

Sahraoui, F., Goldstein, M. L., Robert, P., \& Khotyaintsev, Y. V. 2009, PhRvL, 102, 231102

Salem, C., Mangeney, A., Bale, S. D., \& Veltri, P. 2009, ApJ, 702, 537

Salem, C. S., Howes, G. G., Sundkvist, D., et al. 2012, ApJL, 745, L9

Servidio, S., Osman, K. T., Valentini, F., et al. 2014, ApJL, 781, L27

Servidio, S., Valentini, F., Califano, F., \& Veltri, P. 2012, PhRvL, 108, 045001

Servidio, S., Valentini, F., Perrone, D., et al. 2015, JPIPh, 81, 325810107

Shaikh, D. 2009, MNRAS, 395, 2292

Shaikh, D., \& Shukla, P. K. 2009, PhRvL, 102, 045004

Shaikh, D., \& Zank, G. P. 2009, MNRAS, 400, 1881

Smith, C. W., Hamilton, K., Vasquez, B. J., \& Leamon, R. J. 2006, ApJL, 645, L85

TenBarge, J. M., \& Howes, G. G. 2013, ApJL, 771, L27

TenBarge, J. M., Howes, G. G., \& Dorland, W. 2013, ApJ, 774, 139

Tessein, J. A., Smith, C. W., MacBride, B. T., et al. 2009, ApJ, 692, 684

Tu, C.-Y., \& Marsch, E. 1995, SSRv, 73, 1

Valentini, F., Servidio, S., Perrone, D., et al. 2014, PhPl, 21, 082307 
Vasquez, B. J. 2015, ApJ, 806, 33

Vasquez, B. J., \& Markovskii, S. A. 2012, ApJ, 747, 19

Vasquez, B. J., Markovskii, S. A., \& Chandran, B. D. G. 2014, ApJ, 788, 178

Verdini, A., \& Grappin, R. 2012, PhRvL, 109, 025004

Verscharen, D., Marsch, E., Motschmann, U., \& Müller, J. 2012, PhPl, 19, 022305
Wan, M., Matthaeus, W. H., Roytershteyn, V., et al. 2015, PhRvL, 114, 175002

Wan, M., Perri, S., Osman, K., et al. 2012, PhRvL, 109, 195001

Wicks, R. T., Horbury, T. S., Chen, C. H. K., \& Schekochihin, A. A. 2011, PhRvL, 106, 045001

Wu, P., et al. 2013, ApJL, 763, L30 